\title{
Dante e il prog italiano
}

\author{
Marco Berisso \\ Università di Genova \\ mberisso@unige.it \\ https://orcid.org/0000-0002-3104-2057
}

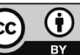

\section{Riassunto}

L'articolo investiga gli espliciti riferimenti alla Commedia dantesca che hanno attraversato la produzione musicale cosiddetta progressive italiana. L'analisi è centrata soprattutto sui rapporti tra il testo dantesco e i testi dei dischi presi in esame, cercando di individuare le dinamiche di riuso dei versi del poema (si va dalla citazione letterale a semplici suggestioni di tipo strutturale) in un arco temporale che parte dal primo accenno a Dante nel disco $U t$ dei New Trolls (1972) per arrivare agli ultimissimi anni (l'ultimo disco di cui si parla, Il Pozzo dei Giganti dei Cherry Five è del 2015).

Parole chiave: rock progressivo; citazione; attualizzazione; intertestualità.

\begin{abstract}
The essay investigates the explicit references to Dante's Commedia that can be found in the so-called Italian progressive music production. The analysis is centered above all on the relationships between Dante's poem and the lyrics of the musical works examined, trying to identify the dynamics of reuse of the verses of the poem, from literal quotation to structure's suggestions. The period involved in the study starts from the first mention of Dante in New Trolls' Ut (1972) since the very latest years.
\end{abstract}

Key Words: progressive rock; quotation; actualization; intertextuality. 
Once there was a man who loved a woman too much

To give up hope when he saw she wouldn't touch him with a barge pole.

He spent his whole life in the Inferno

He composed in thirty-four cantos.

O Dante, though I'm anti such romantic speculation

I'm your hypocrite reader in the same situation,

I'm your double, oh me I'm your brother in pain.

But Alighieri if you'll listen there's a difference

Between your Beatrice and my Paula,

She's anonymous and now a waitress

- It's comic but not divine

The tragedy is no-one's dying!

(Momus, Lucky Like a Saint Sebastian)

$\mathrm{N}$ el quadro complessivo di un'analisi dei rapporti tra l'opera dantesca e la produzione musicale pop italiana ${ }^{\mathrm{I}}$ una fetta consistente riguarda il settore del cosiddetto "rock progressivo" o, più semplicemente (con etichetta mutuata dall'inglese) "prog". Un ruolo non secondario in questo senso ha giocato sicuramente la forte incidenza che questo genere ha avuto in Italia, sia dal punto di vista della ricezione che da quello della produzione: proprio il pubblico italiano, infatti, è stato tra i più accesi sostenitori della musica dei primi gruppi prog anglosassoni, dai Genesis ai Gentle Giant, dagli Emerson, Lake \& Palmer ai Jethro Tull, con un conseguente proliferare di gruppi italiani che ne hanno imitato la produzione e le dinamiche compositive. E dunque la diffusione del genere nella penisola unita alla sua conformazione stilistica (su cui dirò qualcosa schematicamente tra poco) ha ovviamente veicolato, tra le altre cose, un recupero dei testi di Dante e, in particolare, della Commedia.

Le vicende e le caratteristiche del prog sono ben note e ormai ampiamente studiate anche al di là dei testi, diciamo così, 'militanti'. Tra l'altro, nell'ormai vasta bibliografia sul genere ${ }^{2}$ è possibile rinvenire proprio un titolo specifico

I. Preciso che utilizzo "musica pop" nell'accezione originaria, come derivato dall'inglese "popular", a indicare genericamente tutti i fenomeni di musica non-colta. Una categoria che, dal punto di vista strettamente musicale, non ha probabilmente alcun significato: è sufficiente pensare ad esempio a quanti elementi "pop"si incontrano nella composizioni di Mauricio Kagel o, caso addirittura esemplare, di Luciano Berio e, per contro, quanti elementi di musica colta si sono infiltrati nella produzione pop (e il filone di cui qui si discorre ne è proprio uno degli esempi più paradigmatici). La distinzione però credo possa ancora avere una sua validità se riferita alla modalità produttiva e distributiva dei prodotti discografici pop e colti (circuito dei concerti, tipologie di registrazione, modelli di produzione discografica ecc.).

2. Mi limito a citare qui alcuni titoli in stretto ordine cronologico: Mirenzi, 1997; Alfano, 2004; Gaboli e Ottone, 2007; Forni, 2010; Weigel, 2018. Importantissimo anche il volume autoprodotto di Augusto Croce ItalianProg: La guida completa alla musica progressiva italiana degli anni ' 70 , uscito in seconda edizione del 2019; si tratta dell'edizione cartacea, per dire 
che riguarda la questione del rapporto con Dante: mi riferisco alla monografia di Fabrizio Galvagni (Galvagni, 20I2), in cui appunto ritroviamo un'ampia sezione dedicata ai dischi prog italiani. Il volume di Galvagni non esaurisce però del tutto, mi pare, né il panorama né le possibilità di approccio: in parte perché qualche minima cosa era sfuggita alla sua indagine o, per una banale questione di date, non la si era potuta censire (non era stato ancora pubblicato, tanto per dire, Purgatorio dei Metamorfosi che chiudeva nel 2016 la riscrittura/ rielaborazione del poema dantesco avviata con l'Inferno più di quarant'anni prima, nel 1973), in parte perché l'approccio molto appassionato di Galvagni non tocca però direttamente, se non in maniera episodica, l'effettiva consistenza del nesso tra questa produzione musicale e il testo del poema dantesco. Le pagine che seguono si propongono dunque due obiettivi conseguenti a quel che ho appena detto: il primo è quello di integrare le informazioni fornite da Galvagni; il secondo, e credo il più rilevante, è quello di verificare quanto effettivo sia il rapporto con Dante, se cioè ci si trova di fronte a autentiche riprese testuali più o meno precise (se non proprio citazioni dirette) dalla Commedia o se quest'ultima non rappresenti solo un generico richiamo magari di ordine strutturale o immaginativo senza per questo influenzare effettivamente la sostanza verbale delle canzoni o la struttura del disco. ${ }^{3}$

2. Prima di entrare nel discorso specifico, credo sia necessario fornire preliminarmente qualche riferimento cronologico relativo al genere di cui stiamo parlando e indicare quali siano le sue specifiche caratteristiche (o almeno quelle comuni alla maggior parte dei musicisti che ad esso sono solitamente ricondotti).

Come accade sempre per i fenomeni relativi alla musica pop, non solo la data di nascita del rock progressivo ma addirittura quella della stessa definizione è oggetto di discussioni annose. Secondo lo storico statunitense Bradley J. Birzer, il sintagma progressive rock (coniato per analogia con quello progressive jazz già utilizzato a partire dagli anni Venti dello scorso secolo) si ritroverebbe per la prima volta (perlomeno negli USA) nel 1968 in un articolo del «Chicago

così, delle schede contenute nel sito "ItalianProg" (http://www.italianprog.com/), di cui Croce è il curatore e che rappresenta il più importante strumento di informazioni sul genere disponibile in rete (non solo in Italia, peraltro). Altro sito di grande rilievo, raccomandabile soprattutto per il recupero di autori e gruppi scarsamente conosciuti (grazie a un concetto piuttosto ampio di "progressive") di cui vengono messi a disposizione gratuitamente in formato mp3 le registrazioni originali, è il blog "Oltre la stratosfera" (http://verso-la-stratosfera. blogspot.com/). Ricordo infine che dal 2009 viene pubblicata nel Regno Unito la rivista mensile «Prog» di cui esiste, a partire dal 20I5, anche una versione bimestrale italiana (si veda il sito http://www.progressivamente.com/).

3. Premetto che, non avendo specifiche competenze musicali, limiterò i rilievi extratestuali a pochissimi cenni della cui approssimazione mi scuso sin d'ora. 
Tribune»; 4 la versione inglese di Wikipedia indica invece come prima occorrenza quella nelle note interne del primo album omonimo dei Caravan, uscito nel 1969. ${ }^{5}$ Comunque sia, il termine si afferma velocemente e già nel I970-197I appare stabilmente entrato nell'uso del gergo musicale. ${ }^{6}$

In realtà il genere, come spesso accade in casi simili, non nasce dal nulla ma si sviluppa come progressiva estremizzazione di alcuni esperimenti di intreccio tra struttura classica della canzone rock e suggestioni melodiche, ritmiche o strumentali provenienti da altri contesti, come quelli della musica classica (soprattutto nelle sue declinazioni sinfoniche), del folk, del jazz e della musica orientale. Un forte impulso in questa direzione viene ad esempio dalla produzione in studio dei Beatles nella seconda metà degli anni Sessanta, a partire da Rubber soul (1965), in cui brani ancora strutturalmente ben ancorati alla tradizione ${ }^{7}$ ammettono strumenti anomali (il sitar in Norwegian wood, l'harmonium in The word), effetti elettronici (il basso fuzz di Think for yourself) e tecniche innovative di registrazione (il piano elettrico accelerato che nell'assolo centrale di In my life imita la sonorità di un arpsicordo), per passare allo sperimentalismo ancora più pronunciato di Revolver (1966), dove gli elementi derivati dalla musica classica (basti solo pensare alla famosissima Eleanor Rigby, in cui ad accompagnare la voce di McCartney sono esclusivamente due quartetti d'archi) e di manipolazione sonora (i nastri in loop di Tomorrow never knows) sono ulteriormente potenziati, e arrivare infine a Sgt. Pepper's Lonely Hearts Club Band (1967) e al cosiddetto "White Album", il doppio lp The Beatles (1968), dove la costruzione standard della canzone viene ormai sempre più spesso completamente destrutturata. Accanto ai Beatles vengono di frequente ricordati altri gruppi e dischi considerati, per dir così, "protoprog": da Pet Sounds dei Beach Boys (1966) a Mr. Fantasy dei Traffic (1967), dai dischi dei Moody Blues (Days of Future Passed, 1967; In Search of the Lost Chord, 1968) a quelli dei Nice (The Thoughts of Emerlist Davjack, 1967; Ars Longa Vita Brevis, I968) e dei Procol Harum (il singolo A Whiter Shade of Pale, 1967; Shine on Brightly, 1968; A Salty Dog, 1969), tutti caratterizzati da evidenti richiami (se non vere e proprie riscritture) alla musica sinfonica, per arrivare

4. Birzer, 2012.

5. https://en.wikipedia.org/wiki/Progressive rock\#CITEREFMacanI997. Lalbum dei Caravan (formazione riconducibile alla cosiddetta Canterbury Scene) venne pubblicato dalla Verve Forecast (VLP 6oIr; per le informazioni tecniche si veda la scheda sul database Discogs, https://www.discogs.com/it/Caravan-Caravan/release/II70I362)

6. Un'interessante discussione sull'origine del termine corredata da molti materiali documentari (estratti da articoli di giornale, riproduzioni di volantini e manifesti ecc.) è recuperabile nel topic dedicato alla questione dal blog "Prog Archives.com" (http://www.progarchives. com/forum/forum posts.asp?TID $=89567 \& \mathrm{PN}=3$ ).

7. La struttura tipica è quella cosiddetta ISRSRBR, dove I sta per introduzione (di solito strumentale), $S$ per strofa, $R$ per ritornello e $B$ per bridge (ovvero un passaggio che può essere un intermezzo strumentale, un aumento di tono, uno stacco ecc.). 
alla produzione discografica dei Pink Floyd che spesso prelude ad elementi che saranno poi ben presenti nel prog (come accade ad esempio nel secondo lp, quello registrato in studio, del doppio Ummagumma, 1969). Di fatto però, pur essendo il 1969 l'anno di esordio di molti tra i complessi che diventeranno centrali nella successiva stagione progressive, ${ }^{8}$ il disco che viene generalmente considerato fondativo del genere è In the Court of the Crimson King dei King Crimson, pubblicato verso la fine di quell'anno per la Island. ${ }^{9} \mathrm{E}$ proprio basandoci su questo disco possiamo velocemente provare ad elencare le costanti che appunto possono in qualche modo caratterizzare il prog:

I) espansione dell'organico oltre quello tradizionale del rock (formato di norma da chitarra ritmica, chitarra solista, basso e batteria a cui talvolta si aggiungeva un cantante solista), con l'inclusione principalmente di tastiere (in particolare il mellotron, che è in un certo senso lo strumento per eccellenza del prog, e sintetizzatori) e fiati (sassofoni, flauti, clarinetti, ecc.) ma spesso anche di altri strumenti meno usuali (in In the Court of the Crimson King ad esempio si hanno interventi di vibrafono, suonato da Ian McDonald, in Moonchild): questa strumentazione ha spesso un ruolo centrale nell'esecuzione se non addirittura nell'immagine, diciamo così, del gruppo (pensiamo al flauto di Ian Anderson per i Jethro Tull);

2) dilatazione della durata delle singole canzoni che vanno ormai ampiamente oltre i tre minuti canonici (nell'esordio dei King Crimson si va del minimo dei 5' 40" di I Talk to the Wind al massimo dei I2' o9" di Moonchild), per arrivare alle cosiddette "suite", lunghe composizioni dalla struttura complessa destinate ad occupare l'intero lato di un disco; ${ }^{\text {IO }}$

3) potenziamento delle sezioni solo strumentali rispetto a quelle vocali sino alla presenza frequente di brani privi di cantato;

4) maggiore complessità delle strutture ritmiche, armoniche e melodiche, con la conseguente messa in rilievo dell'abilità strumentale dei singoli musicisti;

5) testi che abbandonano le tematiche tradizionali (prevalentemente amorose) per indirizzarsi verso una maggiore elaborazione stilistica, col proliferare di elementi mitici, simbolici, onirici, spesso con riferimenti intertestuali alla poesia colta (non a caso in In the Court of the Crimson King viene accreditato

8. In quell'anno escono infatti il primo disco dei Genesis (From Genesis to Revelation), dei Van Der Graaf Generator (The Aerosol Grey Machine) e gli esordi omonimi degli Yes e dei Renaissance. Esce anche Stand $U_{p}$ (che include la famosa Bourée ripresa da Bach) dei Jethro Tull, che avevano esordito nel I968 con This Was. Sempre al 1968 e poi al i969 datano i primi due dischi dei Soft Machine.

9. Più precisamente il to ottobre (ricorre quindi quest'anno il cinquantennale): scheda in https://www.discogs.com/it/King-Crimson-In-The-Court-Of-The-Crimson-King-AnObservation-By-King-Crimson/release/2287669.

Io. In Thick as a brick dei Jethro Tull (1972) l'unico brano (pure se diviso in sottosezioni) si dilata addirittura sino all'intero lp. 
come membro effettivo del gruppo Pete Sinfield, che non era un musicista ma l'autore delle liriche). ${ }^{\text {II }}$ Questo dato assume un tale rilievo che è assolutamente normale (a differenza di quanto accadeva in precedenza) che nel package del disco vengano riportati i testi delle canzoni;

6) attenzione alla confezione del prodotto discografico, sia dal punto di vista delle copertine (che spesso rappresentano delle vere e proprie esecuzioni grafiche del contenuto musicale e sono affidate a illustratori che si trasformano in un autentico 'marchio visivo' in grado di identificare immediatamente i singoli gruppi ${ }^{12}$ ) che della costruzione dell'oggetto/contenitore (inserimento di veri e proprio libretti all'interno della copertina, forme anomale della stessa, ricorso a vinili colorati anziché neri, ecc.).

La musica prog si diffonde quindi a partire dal 1969 e poi, in maniera sempre più estesa, sino alla metà del decennio successivo, per poi declinare con altrettanta velocità e, per un certo periodo, sparire addirittura del tutto o quasi dalla scena musicale. ${ }^{13}$ Nella vulgata tradizionale il declino del prog viene di solito messo in connessione con il sorgere del punk: ${ }^{\mathrm{I}} \mathrm{e}$ come tutte le vulgate, anche questa contiene una parte di spiegazione autentica ed un'altra, molto più ampia, di semplificazione. ${ }^{\mathrm{IS}}$ Più probabilmente erano in qualche modo le carat-

II. In alcuni casi il testo è scritto addirittura in una lingua inventata. Oltre alla parte centrale del brano The advent of Panurge dei Gentle Giant (da Octopus, I972), ovviamente inserito a emulazione dell'episodio congruente del cap. IX del Pantagruel, va ricordato che i brani del gruppo francese Magma, a partire dal loro primo album omonimo del I970, sono cantati quasi sempre in kobaiano, la lingua che si parlerebbe su quel pianeta Kobaïa le cui vicende sono tema centrale delle loro canzoni Per un minimo approfondimento cfr. il profilo del gruppo ad opera di Peter Thelen (Thelen, I995).

I2. Ad esempio Paul Whitehead, che disegnerà le copertine di tre dischi dei Genesis (Trespass, Nursery Cryme e Foxtrot) e di due dei Van Der Graf Generator ( $H$ to He: Who Am the Only One e Pawn Hearts), oltre che di alcuni dischi degli italiani Le Orme, o Roger Dean, che ha disegnato le copertine di tutti i dischi degli Yes (e di Octopus dei Gentle Giant).

I3. Anche in questo caso sono piuttosto significative le date che indicano cambi nelle formazioni originali delle band o addirittura preludono a lunghi periodi di sospensione dell'attività. Nel 1975 si concludeva il tour in cui i Genesis presentavano The Lamb lies down on Broadway (uscito l'anno prima) e il cantante Peter Gabriel annunciava l'abbandono del gruppo; nel I973, dopo l'ambizioso Tales from a topographic ocean, il tastierista Rick Wakeman lasciava gli Yes; del 1973 è l'ultimo disco propriamente prog dei Gentle Giant, In a glass house; nel I974, subito dopo la pubblicazione di Red, si sciolgono i King Crimson e nello stesso anno, conclusa con You la trilogia Radio Gnome Invisible, interrompono la loro attività anche i Gong. Sempre a partire dal 1974, infine, dopo aver pubblicato il triplo disco dal vivo Welcome back, my friends... che ne compendiava in qualche modo l'attività, i tre componenti degli Emerson, Lake \& Palmer iniziavano di fatto la loro carriera solista.

I4. Nel 1976 escono il 45 giri Anarchy in the UK dei Sex Pistols e il primo lp dei Ramones.

I5. Famoso l'episodio del cantante dei Sex Pistols, Johnny Lydon (noto come Johnny Rotten), che invitato il I6 luglio 1977 a Capitol Radio per il programma The Punk and His Music propose agli ascoltatori una scaletta assolutamente inattesa che includeva anche due brani di Peter Hammill, il cantante dei Van Der Graaf Generator di cui Lydon si dichiarò in quell'occasione un fan entusiasta. E del resto quell'ampio e rilevante fenomeno che è stato il post-punk ha sicuramente assunto più di un elemento dalla musica prog (al punto che Simon Reynolds, autore della più importante monografia su di esso, è arrivato a dire che «In un certo senso il post-punk era progressive rock, solo drasticamente semplificato e 
teristiche stesse del genere (incluse quelle testuali) a trovarsi spiazzate di fronte al sorgere di nuovi soggetti sociali che apparirono sulla scena soprattutto europea a partire dalla metà degli anni Settanta e sino alla fine del decennio. Non è un caso che l'ultima manifestazione che si potrebbe ricondurre alla stagione del rock progressivo sia il cosiddetto "Rock in Opposition" ${ }^{16}$ in cui si assiste al tentativo di unire alla sperimentazione musicale (fattasi per certi versi ancora più estrema) tematiche di aperta critica politica. E però, dopo una pausa che ha attraversato in sostanza tutti gli anni Ottanta e i primissimi anni Novanta, il genere ha conosciuto un momento di decisa riemersione a partire dalla fine del secolo scorso e ancora negli anni Zero-Dieci di questo, riemersione che tra l'altro appare in buona sostanza sganciata dai gruppi storici (ormai quasi tutti inattivi) e legata semmai al manifestarsi di sigle di nuova formazione. ${ }^{17}$

3. Anche le sorti italiane della musica prog hanno seguito più o meno la medesima parabola. I primi dischi abitualmente considerati appartenenti al genere sono Concerto grosso dei New Trolls, Collage delle Orme e L'uomo degli Osanna, pubblicati tutti e tre nel $197 \mathrm{I} .^{\mathrm{I}}{ }^{8} \mathrm{Nel}$ giro di pochissimi anni si moltiplicarono i gruppi e le incisioni, al punto che le caratteristiche del genere finirono per essere assunte anche da quell'altro fenomeno tipicamente italiano ma apparentemente antitetico, che è stato la musica cantautorale. ${ }^{19}$ Come anticipavo, non può stupire, date le caratteristiche del genere, che alcuni gruppi abbiano finito con l'incrociare in qualche modo la Commedia, da cui hanno derivato principalmente, come vedremo tra poco, o suggestioni di tipo simbolico e

rinvigorito, con acconciature migliori e una sensibilità più austera»; cfr. Reynolds, 20Io, p. XXI).

I6. La definizione deriva dall'omonimo concerto organizzato il I2 marzo I978 a Londra e a cui parteciparono cinque gruppi provenienti da altrettante nazioni (gli inglesi Henry Cow, i belga Univers Zero, i francesi Etron Fou Leloublan, gli svedesi Samla Mammas Manna e gli italiani Stormy Six). In realtà concerti sotto l'etichetta "Rock in Opposition" (spesso siglato RIO) sono stati organizzati ancora negli ultimissimi anni (l'ultimo si è tenuto in Francia dal I4 al I6 settembre 20I8: il programma è all'indirizzo http://rockinopposition.rocktime. org/index.php/en). Per un profilo sintetico del movimento si veda la scheda dedicatagli sul sito Prog Archives.com (https://www.progarchives.com/subgenre.asp?style=36).

17. Per un quadro generale sul cosiddetto neo-prog cfr. Weigel, 20I8, pp. 217-7I. Fa eccezione a quanto detto in precedenza proprio l'Italia, dove negli ultimi dieci/quindici anni non sono mancate reunion (dagli Osanna al Banco di Mutuo Soccorso, dai Pholas Dactylus ai Rovescio della Medaglia al Balletto di Bronzo), magari con qualche cambio nei componenti originari, che hanno spesso portato all'incisione di nuovi dischi persino in questo 2019 (a maggio è stato pubblicato Transiberiana del Banco di Mutuo Soccorso, a giugno Metafisica degli Alluminogeni e Hieros Gamos dei Pholas Dactylus ecc.). Imprescindibile, comunque, per un quadro degli sviluppi del genere in Italia a partire dagli anni Ottanta è Salari, 2018 (si noti che delle circa trecento pagine di gruppi lì censiti più o meno due terzi sono riservate a quelli che hanno iniziato la propria carriera musicale proprio negli anni Duemila).

I8. Una rilevante documentazione sulla trasformazione che ha portato in Italia la musica beat della seconda metà degli anni Sessanta alla sua evoluzione verso il prog (spesso con il coinvolgimento delle medesime formazioni) è in Marino e Bruno, 2015.

I9. Sul fenomeno cfr. Pardo, 2017, pp. 26-37. 
fantastico o un'impostazione che potremmo dire latamente militante, magari riattualizzando il testo di Dante in maniera più o meno netta. Per contro, rimangono ovviamente del tutto esclusi da queste produzioni sia lo sfondo storico quanto gli elementi propriamente dottrinali. Va anche aggiunto che, per quanto ciò possa apparire contraddittorio per autori che derivano il proprio stile dalla musica anglosassone, è forse percepibile una sorta di nazionalismo che può aver rindirizzato verso un Dante "padre della lingua" di ascendenza addirittura risorgimentale. A sostegno di quanto ho appena osservato ricordo che all'interno della copertina del disco Ut dei New Trolls (1972) veniva riportato il famoso passo iniziale dell'undicesimo capitolo del Convivio (I xi I-2) in cui Dante depreca coloro che deprezzano il proprio volgare. ${ }^{20} \mathrm{Il}$ riferimento è ovviamente tutt'altro che casuale e allude infatti alle vicissitudini interne del gruppo genovese, che sempre nel 1972 aveva pubblicato il proprio unico album interamente cantato in inglese Searching for a land: Ut sarebbe quindi in un certo senso da intendersi come una palinodia di Searching for a land, visto che i testi sono questa volta tutti in italiano. ${ }^{2 \mathrm{I}}$

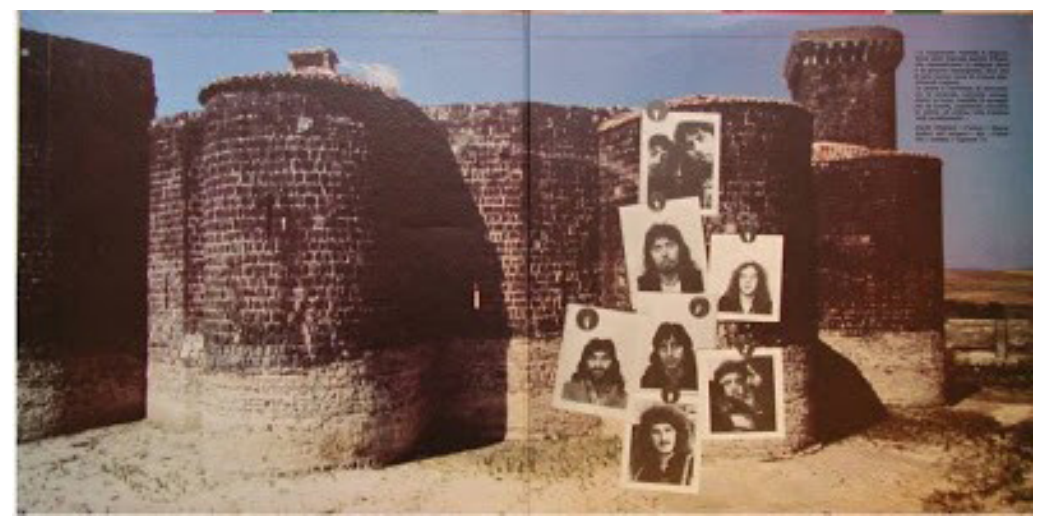

Fig. I Interno della copertina di Ut dei New Trolls con la citazione dal Convivio.

20. «A perpetuale infamia e depressione delli malvagi uomini d'Italia, che commendano lo volgare altrui e lo loro propio dispregiano, dico che la loro mossa viene da cinque abominevoli cagioni. La prima è cechitade di discrezione; la seconda, maliziata escusazione; la terza, cupidità di vanagloria; la quarta, argomento d'invidia; la quinta e l'ultima, viltà d'animo, cioè pusillanimità». Galvagni 2012 (p. 58), fa tra l'altro notare come la copertina del disco presenta non per caso i tre colori della bandiera italiana.

2I. Curiosamente, però, il disco prende il titolo dal modo con cui comunemente viene definito il do nella cultura musicale anglosassone ( $u$ t, appunto). Sulle non linearissime vicende dei New Trolls, passati attraverso rimescolamenti di formazioni e creazione di formazioni parallele, si può vedere la scheda riassuntiva del sito Italian Prog http://www.italianprog. com/it/a newtrolls.htm. 


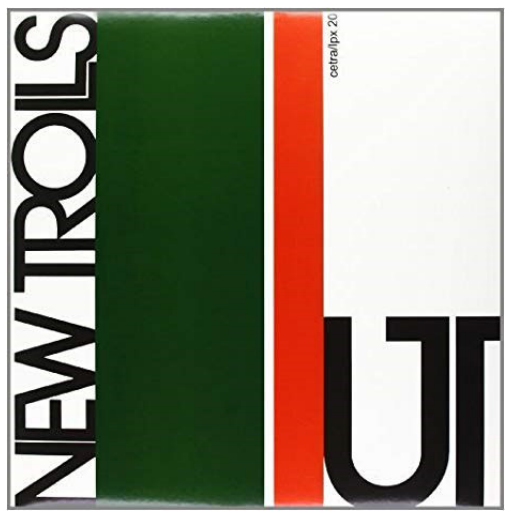

Fig. 2 Copertina di Ut dei New Trolls

Non sorprende allora che in questo disco nato sotto una così visibile impronta dantesca si possa ritrovare una canzone intitolata Paolo e Francesca. Si tratta di una ballad per molti aspetti tradizionale, caratterizzata soprattutto dal ricorso ad una chitarra elettrica (suonata da Nico Di Palo) che grazie all'uso del wah wah e della leva del tremolo imita, nella coda strumentale, una specie di conversazione tra due persone. Il brevissimo testo ${ }^{22}$ utilizza l'episodio dantesco come proiezione e parallelo per un'altra storia amorosa contemporanea. Il rinvio all'episodio di Paolo e Francesca permette di capire che ciò a cui si allude nella canzone è un adulterio («Il loro peccato è sospeso su noi») ed è proprio e solo in questo che si esaurisce nella sostanza il riferimento dantesco (evocato però esplicitamente nel testo in funzione, diciamo così, autoriale: «La storia ci dice è stato così). Infatti, a parte sporadici e neppure sicuri echi lessicali, nessun elemento connotato del pur memorabile episodio viene trasportato nella canzone. ${ }^{23}$

4. Seguendo il procedere cronologico, la prima produzione italiana (ma in senso largo, come vedremo subito) riconducibile al prog ad aver assunto Dante

22. Si tratta di quattro gruppi (due utilizzati per le strofe e due come ritornello) di quattro versi costituiti nella maggior parte dei casi dalle stesse frasi con minime variazioni. Le note di copertina non accreditano direttamente nessuno dei componenti del gruppo per la parte testuale: gli autori di Paolo e Francesca sono Nico Di Palo, Gianni Belleno, Rhodes (pseudonimo del bassista Frank Laugelli) e Stefano Chiabrera, violoncellista già apparso in Concerto Grosso.

23. Anzi, proprio la prima strofa ( Tu cerchi sempre Paolo / Francesca cerca te / Nel cielo senza nuvole / La strada che non c'è»), dove pure si evoca il vagare dei due personaggi nel cielo infernale (il "cielo senza nuvole» sarà traccia dell' «aere maligno» e dell' "aere perso" che si susseguono a brevissima distanza in If. V 86 e 89?), si distacca dal più significativo degli elementi danteschi, ovvero il fatto che Paolo e Francesca siano uniti eternamente nella pena come lo furono nella vita (il riferimento è ovviamente al famoso «questi, che mai da me non fia diviso", If. $\mathrm{V}_{\text {I35). }}$. 
come riferimento per un proprio disco sono stati i The Trip. Italiana in senso largo, dicevo, perché il gruppo era in realtà formato per metà da musicisti inglesi (Billy Gray, chitarra e voce, e Arvid Andersen, basso e voce) e per l'altra metà italiani (Joe Vescovi, tastiere e voce, e Pino Sinnone, batteria). Dopo l'esordio omonimo nel 1970, i The Trip producono nel 1971 il loro secondo disco intitolato Caronte. Si tratta di quello che viene definito un concept album, un disco, cioè, in cui le singole canzoni sono collegate tra loro in maniera narrativa o tematica, spesso con recuperi di un medesimo tema melodico in punti diversi del disco: una costruzione che, come si capisce, si addice bene alle ambizioni 'operistiche' della musica prog. ${ }^{24} \mathrm{Nel}$ caso dei The Trip il tema attorno a cui ruota il disco è appunto la figura di Caronte, traghettatore infernale che viene però considerato come una sorta di guida (trasformandolo, diciamo così, in un Virgilio) per affrontare un viaggio verso un aldilà in cui si trovano, nell'ordine, le anime dannate di due fratelli deceduti in un incidente stradale, quella di Janis Joplin e quella di Jimi Hendrix, questi due morti entrambi l'anno prima e diventati immediatamente figure iconiche della musica e cultura pop. La costruzione del disco si articola quindi intorno a cinque brani, due strumentali, posti rispettivamente in apertura e in chiusura e che rielaborano lo stesso tema musicale come appunto richiesto dalla struttura del concept (Caronte I e Caronte II), e tre cantati centrali (Two brothers, Little Jeanie e L'ultima ora e Ode a Jimi Hendrix). La coesione dell'insieme è data appunto da questa specie di cornice para-narrativa a cui si riferisce anche la nota rimata interna al disco (scritta in un inglese a dir poco bizzarro) che qui riporto: ${ }^{25}$

Dear Charon, thank you for the invitation

to look apon a souls damnation.

With us are a chosen few

that we should like to interview.

First the two of steel and leather

that met a violent end together.

Then the tale of Janies parting

that put an end to all her tarting.

And was the dearth of dearest Jimi

brought apon by Micky Finny.

So leave it to imagination

to be the source of your creation.

24. Cfr. Follero, 2009 (in particolare per Caronte cfr. pp. I36-I38). Cfr. anche Galvagni, 20I2, pp. 53-54.

25. Inserisco io la punteggiatura ma mantengo tutto il resto alla lettera, incluso l'inaudito e doppio apon, che mi fa supporre che ci si trovi di fronte alla trascrizione ad opera di qualcuno con non perfette competenze in inglese di un passo dettato ad alta voce. Per la comprensione si tenga conto che Micky Finny (in realtà Mickey Finn) indicava in gergo una bevanda alcolica a cui fosse stata aggiunta una qualche sostanza psicotropa. 


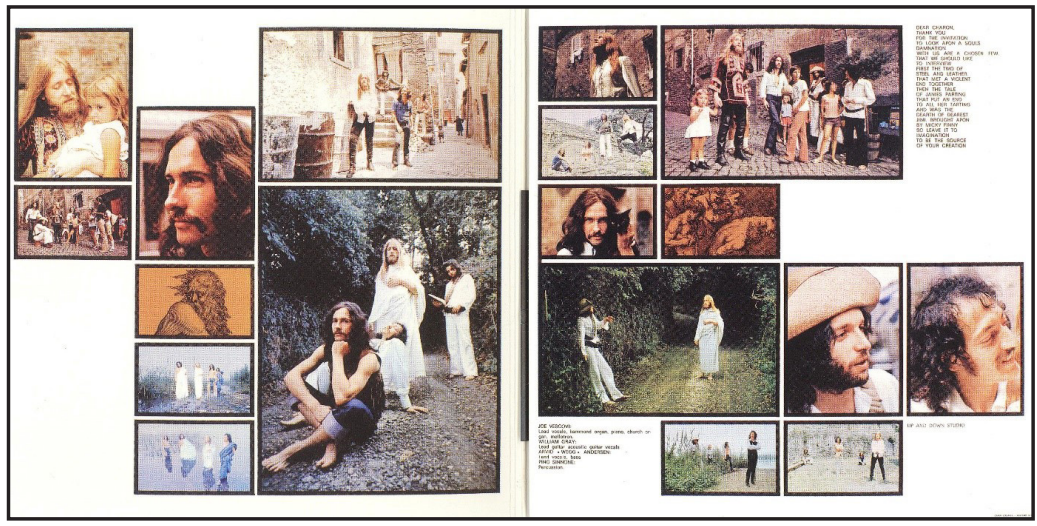

Fig. 3 Interno della copertina di Caronte dei The Trip

I testi, scritti da Gray, sono tutti in inglese (anche quello di L'ultima ora e Ode a Jimi Hendrix, nonostante il titolo) e non presentano riferimenti evidenti a Dante. L'unico collegamento palese tra il disco e il poema e quello che ne giustifica la menzione in queste pagine è legato in sostanza alla copertina del disco. Su di essa troviamo infatti la rielaborazione di due famose illustrazioni di Gustav Dorè a If. III dedicate appunto a Caronte, la prima relativa ai vv. 82-84 («Ed ecco verso noi venir per nave / un vecchio, bianco per antico pelo, / gridando: "Guai a voi, anime prave!"»), la seconda ai vv. II5-II6 («similemente il mal seme d'Adamo / gittansi di quel lito ad una ad una»).
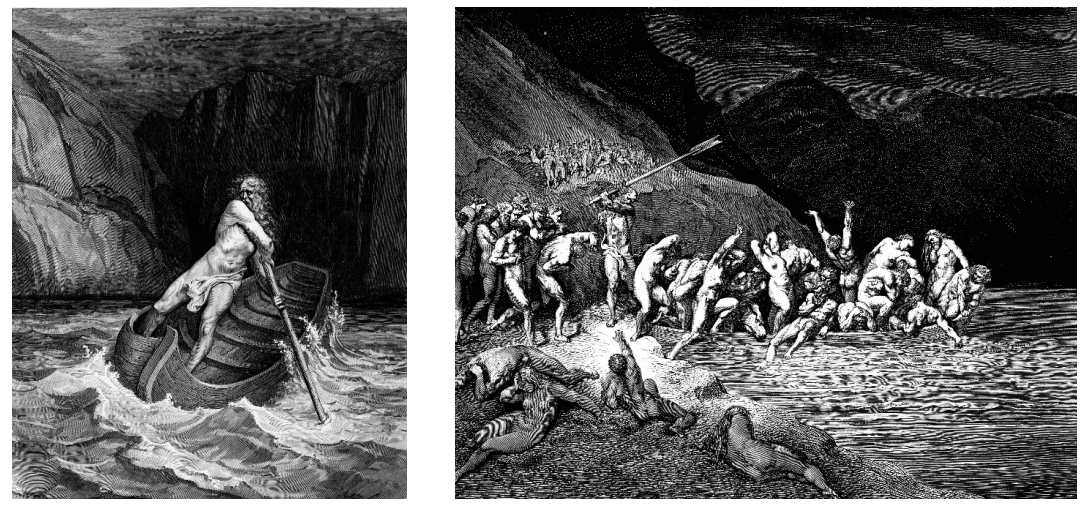

Figg. 4-5 Illustrazioni di Gustav Doré a If. III. 
Le due immagini sono riprese nella copertina di Caronte, rispettivamente sul recto e sul verso, con l'aggiunta di elementi che dovrebbero in qualche modo riattualizzarne la costruzione. Così la bandiera inglese (a ricordare appunto la provenienza di metà dei componenti del gruppo) è sia collocata al posto del drappo bianco intorno alla vita del nocchiere sia posta sul retro a cammuffarne il remo. L'origine italiana dell'altra metà del gruppo è invece suggerita dai colori del biplano presente sempre sul retro di copertina. Altri elementi di questa stessa immagine potrebbero avere un significato parodico (il costume da bagno della dannata sulla barca di Caronte, il nome del gruppo scritto su un cartello retto da un altro dannato ecc.), anche se non saprei dire quanto volontario, mentre ad un gusto genericamente psichedelico sono da ricondursi tanto il cielo giallo quanto i rami che appaiono qui e là (oltre, ovviamente, ai disegni astratti che circondano la foto dei componenti del gruppo).

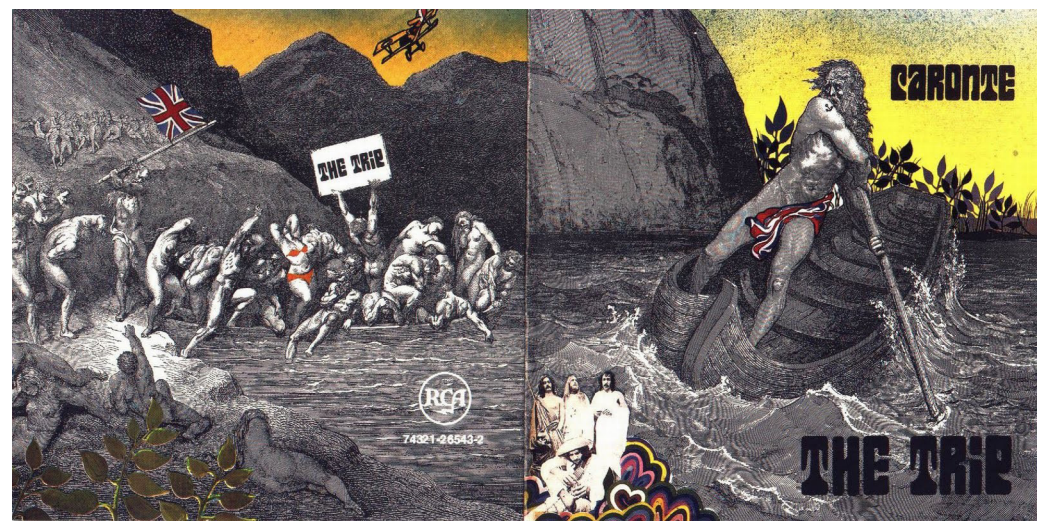

Fig. 6 Copertina di Caronte dei The Trip.

Nel complesso comunque la rielaborazione grafica della copertina funziona come esplicito invito per l'ascoltatore (l'unico, dicevo, ma evidentemente molto forte) a collegare il disco alla Commedia e dunque chiarisce simmetricamente, per dire così, anche l'operazione musicale complessiva, che si propone cioè nei termini di un riammodernamento della struttura (in senso largo) del poema dantesco. Nello stesso tempo è però evidente che tale struttura rimane sostanzialmente un corpo esterno, se non proprio estraneo, rispetto alle canzoni in quanto tali. Caronte utilizza insomma il poema solo come suggestione generica e come modello culturale, senza che questo implichi di necessità un ricorso diretto ed effettivo ad esso. ${ }^{26}$

26. Segnalo incidentalmente che nel 2016 è uscito per l'etichetta A Simple Lunch un disco intitolato Caronte (tra Calabria e Sicilia) opera del musicista (e astronomo) Angelo Adamo (se ne veda la presentazione d'autore sul sito della casa discografica, https://www.asimple- 
5. Un semplice cenno, nonostante il titolo appaia allettante, merita il disco La Divina Commedia de Il Giro Strano. Originari di Savona, Il Giro Strano sono uno dei moltissimi gruppi che nella prima metà degli anni Settanta parteciparono ai frequentissimi festival musicali organizzati in tutta Italia ma che poi, per varie ragioni, non arrivarono mai ad incidere un disco prima dello scioglimento. La Divina Commedia, pure se basato su registrazioni databili al 1972-1973, esce infatti per la Mellow Records solo nel 1992, quasi dieci anni dopo la morte del cantante Mirko Ostinet, avvenuta nel 1983. ${ }^{27}$ Come spesso capita in casi simili (tutt'altro che infrequenti, sia detto tra parentesi), la qualità di queste registrazioni è variabile e, in alcuni casi, bassissima. È proprio quest'ultimo il caso del lungo brano (oltre quindici minuti) che dà il titolo all'album, evidentemente registrato durante una sessione di prova. Le note di copertina lo segnalano come suddiviso in quattro sezioni (Inferno, A Riveder Le Stelle, Purgatorio e Paradiso) ma è difficile all'ascolto avere una percezione precisa di tali partizioni interne. La Divina Commedia è per la sua maggior parte un pezzo solo strumentale ${ }^{28}$ ad eccezione di una sezione verso la fine (e quindi presumibilmente corrispondente al Paradiso) cantata in inglese ma il cui testo è nella sostanza incomprensibile a causa appunto del livello tecnico dell'incisione. Resta il fatto che anche in questo caso, come per i The Trip, l'influenza dantesca sembrerebbe da circoscrivere ad un elemento di pura suggestione strutturale senza rapporti diretti con il testo del poema.

Il primo gruppo prog italiano per cui sia possibile quindi spendere effettivamente e a pieno diritto il nome di Dante è Metamorfosi, band romana autrice di una trilogia dedicata alla Commedia, aperta con Inferno (1973), proseguita con Paradiso (2004) e chiusa da Purgatorio (2016). Come si vede dalle date, in realtà proprio dopo la pubblicazione di Inferno il gruppo si sciolse e le due incisioni più tarde sono opera di una formazione solo in parte coincidente con quella originale: ${ }^{29}$ e anche la struttura dei singoli dischi e la costruzione

lunch.com/it-IT/component/k2/item/64-caronte-tra-calabria-e-sicilia.html): si tratta di un album di grandissimo interesse, centrato sulla rielaborazione di atmosfere folk, ma non riconducibile al prog. Il riferimento a Caronte contenuto nel titolo, come si spiega nella scheda online che ho appena citato, è da interpretare come allusione al traghetto che unisce appunto la Calabria alla Sicilia ( Se davvero si vuole abitare per una manciata di minuti questi tredici scorci di un unico paese diviso e unito da una stretta lingua, una lingua di mare, ed esistente in un meta-livello tra realtà, sogno e ricordo, sarò felice di traghettarvi da una sua sponda all'altra narrandovene la mitologia») e quindi non pare collegarsi direttamente alla Commedia.

27. Per la scheda tecnica del disco cfr. https://www.discogs.com/it/Il-Giro-Strano-La-DivinaCommedia/release/3581016. Un breve profilo del gruppo in http://www.italianprog.com/ it/a girostrano.htm.

28. E, sia detto per inciso, tutt'altro che disprezzabile, soprattutto nelle partiture di hammond e di sax tenore suonate rispettivamente da Alessio Feltri e Mariano Maio.

29. In Inferno troviamo Roberto Turbitosi al basso e alla voce, Gianluca Heygers alla batteria e percussioni, Enrico Olivieri alle tastiere, pianoforte e voci e Jimmy Spitaleri alla voce solista e al flauto; della formazione originaria in Paradiso e Purgatorio restano Olivieri e Spitaleri 
dei testi in rapporto a Dante ha subito nel corso dei decenni, come vedremo, delle decise trasformazioni.

Partiamo allora da Inferno..$^{\circ} \mathrm{Il}$ disco si struttura in due suite, una per facciata, a loro volta articolate rispettivamente in nove e sette parti senza soluzione di continuità tra di loro. Il legame tra le due suite è dato (secondo una procedura usuale all'epoca) dalla ripresa all'inizio del lato B in fade-in del medesimo motivo musicale su cui si era chiuso in fade-out il lato A (nel caso specifico, una serie di accordi di organo)..$^{3 \mathrm{I}}$ Questo è l'elenco delle sezioni, divise per lato:

Lato A
Introduzione
Selva Oscura
Porta dell'Inferno
Caronte
Spacciatore di Droga
Terremoto
Limbo
Lussuriosi
Avari

Lato B

Violenti

Malebolge

Sfruttatori

Razzisti

Fossa dei Giganti

Lucifero (Politicanti)

Conclusione

Avari

Come si può notare, se si bada solo ai "peccati" puniti in questo Inferno riaggiornato (Spacciatore di Droga, Lussuriosi, Avari, Violenti, Sfruttatori, Razzisti e Politicanti) e si addizionano le zone propriamente desunte da Dante del Limbo e di Malebolge si ottiene un numero di ipotetici gironi esattamente corrispondente a quello della Commedia. Allo stesso modo è congruente alla collocazione nel poema il posizionamento del Pozzo dei Giganti (qui Fossa dei Giganti) prima dell'ultimo girone. Per il resto, però, la topografia infernale dei Metamorfosi rielabora quella originaria con estrema libertà: basti pensare al posizionamento di Malebolge e a quello del Limbo, completamente sfalsati. Più aderente, semmai, la sequenza (pur con inevitabili salti) della serie Lussuriosi, Avari e Violenti che corrisponde grosso modo a quella originaria (sarebbero i cerchi II, IV e VII di Dante). Fatti i distinguo necessari e segnalate le incongruenze (che però sono comprensibili, dato l'approccio ovviamente non filologico alla fonte), resta comunque evidente la volontà di fornire all'ascoltatore una

mentre al basso suona Leonardo Gallucci e alla batteria Fabio Moresco (in Paradiso Oliveri è affiancato alle tastiere da Marco Morcacci).

30. Per la scheda tecnica cfr. https://www.discogs.com/it/Metamorfosi-Inferno/release/38I69io.

3I. Può non essere inutile ricordare che per tutti questi musicisti, perlomeno all'altezza della loro produzione degli anni Settanta, l'unità base entro cui strutturare il materiale non era l'intero disco ma il singolo lato in cui il lp in vinile si articolava. Questo comportava, appunto, la necessità di artifici di connessione nei casi, come questo, in cui l'idea da restituire fosse quella "poematica" di un unico brano (o di una serie strettamente collegata di brani) che occupasse l'intero disco. 
struttura che evochi in maniera più o meno esatta la memoria della Commedia e allo stesso tempo restituisca, grazie anche all'assenza di fratture nel continuum musicale, l'idea di un percorso e quindi, come nel poema, di un viaggio.

Passando alle singole sezioni del disco, ed escludendo le strumentali Selva Oscura, Terremoto, Limbo e Fossa dei Giganti, ${ }^{32}$ sono strutturati in forma di dialogo i testi ${ }^{33}$ di Lussuriosi e Avari mentre gli altri sono organizzati in forma monologica come discorsi rivolti direttamente ai dannati di volta in volta chiamati in causa. Manca di norma ogni descrizione delle pene a cui le anime dovrebbero essere sottoposte (ad eccezione dei razzisti: «infissis a queste croci / adesso voi bruciate»), mentre la modalità elocutiva di fondo, ovvero quella dell'invettiva, finisce col declinarsi spesso nei termini di un moralismo parareligioso che credo risponda allo spirito del gruppo più ancora che a quello dei tempi ${ }^{34}$ e che è, comunque, una quasi inevitabile ricaduta di quella attualizzazione di cui si diceva.

Non sorprende a questo punto che la presenza di espliciti intertesti dalla Commedia nelle singole liriche sia piuttosto discontinua né che essa si concentri soprattutto nei brani non esplicitamente dedicati ai singoli dannati. Così, se in Introduzione i versi «alberi tristi tendono al cielo / rami corrosi dal tempo» sono una rielaborazione dell'incipitaria selva oscura dantesca, i due brevissimi testi di Porta dell'inferno e Caronte sono evidentemente ricalcati sui corrispettivi passi di If. III. ${ }^{35}$ Un vago riferimento potrebbe essere recuperato anche all'inizio di Spacciatore di droga ("Ora che imprechi per la rabbia ed il dolore») visto che il possibile intertesto (penso al v. 26 "parole di dolore,

32. Fossa dei Giganti ha però una evidente funzione di prologo per la sezione successiva, Lucifero (Politicanti), visto che al suo interno vengono accennati dal sintetizzatore prima l'inno nazionale degli Stati Uniti e poi quello dell'Unione Sovietica e che proprio i presidenti di quelle due nazioni erano, secondo le dichiarazioni di Spitaleri, oggetto di Lucifero («Il nostro "Inferno" condannava la divisione del mondo in blocchi contrapposti, con il duo dominante Stati Uniti-Unione Sovietica che decideva i destini del mondo. Proprio in un brano, esplicitamente, facevamo maciullare i loro leader da Lucifero (allora c'erano Johnson e Nixon negli Usa e Breznev a Mosca)»: così il cantante nell'intervista rilasciata nel dicembre 2009 alla rivista online «PlanetRock», http://www.pianetarock.it/intervista oo6metamorfosi.asp).

33. Tutti tendenzialmente molto brevi.

34. Pur senza voler insistere troppo su questo, va però ricordato che i Frammenti, vale a dire il nucleo originario da cui scaturirono i Metamorfosi, erano specializzati nel genere delle cosiddette "messe beat" (si veda la già citata intervista a Spitaleri) e tematiche religiose erano ben evidenti anche nel primo disco del gruppo ...E fu il sesto giorno (1972).

35. In Porta dell'Inferno, oltre alla ovvia riproposta del famoso v. 9 («Lasciate ogni speranza / o voi che entrate»), si trova anche un'evidente calco proprio dall'episodio di Caronte («anime dannate / al caldo e al gelo soffrirete» rinvia evidentemente a III 87: «ne le tenebre etterne in caldo e 'n gelo»). Altrettanto precisi i recuperi in Caronte, da If. III Io9 ("Caronte demonio / occhi di fuoco nel buio») e 85 ( E non sperate mai di rivedere il cielo»). Pur mettendo in conto una certa lessicalizzazione 'infernale' non necessariamente da ricondurre a Dante, segnalo che anche nell'ultimo verso di Caronte ("anime nere al fuoco eterno brucerete») si possono recuperare memorie tanto del «foco etterno» di If. VIII 73 quanto (e soprattutto) di If. VI 85: "Ei son tra l'anime più nere». 
accenti d'ira») si trova ancora nell'orbita del terzo canto, mentre un accenno all'episodio di Paolo e Francesca (che, come si vedrà, è il più frequentato da questi musicisti ${ }^{36}$ ) può essere recuperato all'inizio di Lussuriosi ( Siamo dannati insieme») e un ricordo del Flegetonte dantesco nell'avvio di Violenti («Rosso scorre il sangue dai sentieri / dov'è fragile la vita»: ma potremmo trovarci di fronte ad una banale metaforizzazione ${ }^{37}$ ). Nessun riferimento diretto invece, un po' sorprendentemente, è dato cogliere in Malebolge, ${ }^{38}$ mentre in Lucifero (Politicanti) sono per contro evidenti i richiami ai canti conclusivi del poema, dall'evocazione di Cocito ( Immersi in questo mare / voi gelerete in eterno») al preciso rinvio a If. XXXIV 55-56 («Da ogne bocca dirompea co' denti / un peccatore, a guisa di maciulla») nella descrizione di Lucifero («maciulli quei dannati / sfogando la tua rabbia»). Infine in Conclusione troviamo, come era da attendersi, una rielaborazione del verso finale della prima cantica ( $\mathrm{E}$ fu così che noi tornammo a riveder le stelle»). Nel complesso, come si può vedere, i richiami espliciti a Dante vanno a toccare canti e versi notissimi, secondo un procedimento abituale in questo genere di operazioni che richiedono in sostanza, per essere condivise, una forma di immediato riconoscimento da parte del loro pubblico. Ha comunque giocato di sicuro un ruolo centrale in questo senso il fatto che l'Inferno sia la cantica tradizionalmente più frequentata e memorizzata anche a livello scolastico e dunque la più immediatamente disponibile alla memoria e all'immaginario degli ascoltatori.

Se passiamo a Paradiso ${ }^{39}$ (conviene ovviamente seguire l'ordine di pubblicazione degli album piuttosto che quello delle cantiche), possiamo verificare alcune innovazioni rilevanti rispetto a Inferno già in relazione alla struttura del disco..$^{\circ}$ La prima novità è che le sezioni non sono più strettamente collegate tra loro in un' unica suite (o in due suite connesse) come nella prima cantica ma si presentano spesso, più tradizionalmente, come singoli pezzi o, in alternativa, come brevi sequenze autonome,${ }^{41}$ ma comunque con una perdita del

36. E non solo: alcuni esempi (da De Andrè, Venditti, Jovanotti) in Galvagni, 20I2, pp 89-93. Più in generale per il rapporto tra Dante e la produzione cantautorale cfr. Francesco Ciabattoni, Dante and Italy's Singer-Songwriters, in "Italian Quarterly», 54 (2017), pp. 61-80.

37. Tra parentesi, segnalo che proprio questo brano, in cui si rievoca un omicidio di mafia, è probabilmente l'unico in cui la procedura di riattualizzazione sia condotta con una certa efficacia.

38. Altamente improbabile, per quanto invitante, è pensare che in un verso come «buio di una notte senza fine» possa ritrovarsi traccia del noto avvio di Pg. XVI I-2: «Buio d'inferno e di notte privata / d'ogne pianeto».

39. Per la scheda tecnica del disco cfr. https://www.discogs.com/it/Metamorfosi-Paradiso/master/I549042.

40. Andrà segnalata, in primo luogo, la novità tecnica del passaggio del supporto dal vinile al $\mathrm{cd}$, che ovviamente non prevede la spezzatura del materiale musicale in due parti. In questo caso, però, non mi pare che questo abbia influenzato la composizione (nemmeno per quel che riguarda la durata, che supera solo di poco i quarantacinque minuti canonici).

4I. Ad esempio sugli armonici dell'accordo finale di pianoforte di Sfera di fuoco si innesta 
carattere metaforicamente poematico della parte musicale (e anche, quindi, di quel viaggio che come dicevo questo carattere suggeriva). Altro elemento notevole, a fronte di una proporzione tra brani cantati e brani strumentali più o meno equivalente a quella di Inferno (quattro su quattordici), è la decisa espansione della parte testuale, che nel primo disco era solitamente composta da pochi versi. Vediamo comunque, come in precedenza, la successione dei brani:

\author{
Introduzione \\ Sfera di fuoco \\ Cielo della Luna \\ Salita a Mercurio \\ Cielo di Mercurio \\ Salita a Venere \\ Cielo di Venere (Notturno su Venere) \\ Il Sole \\ Cielo di Marte \\ Cielo di Giove \\ Cielo di Saturno \\ Stelle Fisse \\ Empireo \\ La Chiesa delle Stelle
}

Come si può notare i pezzi si intitolano ai singoli cieli danteschi rispettandone la successione e il numero (ad eccezione del Primo Mobile che viene identificato evidentemente con l'Empireo): in due casi (per Mercurio e per Venere) viene poi segnalato un elemento narrativo ben presente nella Commedia, vale a dire la descrizione dell'ascesa da un cielo a quello successivo. Il carattere puramente denotativo dei titoli (di contro a quello tutto connotativo dei corrispondenti di Inferno) conosce solo due infrazioni poste ai due estremi della sequenza (tenendo da parte, come strutturalmente ovvio, Introduzione), ossia Sfera di fuoco e La Chiesa delle Stelle. La distribuzione dei pezzi strumentali non pare invece possedere un particolare valore strutturale. ${ }^{42} \mathrm{Nel}$ complesso, quindi, l'adesione alla fonte è da questo punto di vista molto più marcata rispetto al disco precedente. Per quel che riguarda i singoli testi, il rapporto con la Commedia si articola nei termini di un sostanziale rispetto della struttura

l'arpeggio sempre di pianoforte iniziale in Cielo della Luna, che a sua volta si conclude con un accordo di tastiera elettronica da cui prende avvio il pianoforte di Salita a Mercurio (è lo stesso arpeggio di Cielo della Luna alzato di tono). La continuità evidentemente intenzionale di questa sequenza prosegue con Cielo di Mercurio, uno strumentale sempre avviato dal pianoforte, e lì si interrompe, visto che il successivo Salita a Venere è separato da uno stacco di silenzio. Collegate risultano anche Cielo di Marte e Cielo di Giove e (meno accentuatamente) la coppia conclusiva Empireo e La Chiesa delle Stelle.

42. Sono strumentali Cielo di Mercurio, Salita a Venere (questi due subito in successione), Il Sole e Empireo. 
discorsiva dei singoli canti o gruppi di canti (cercherò tra poco di spiegare meglio in che senso) che vengono però quasi sempre risemantizzati in chiave lirico-simbolica. ${ }^{43} \mathrm{E}$ sufficiente in questo senso analizzare le prime composizioni del disco. Partiamo da Sfera di fuoco:

Luce intensa di fuoco

fugge il luogo e va verso la terra,

spegne gli occhi di chi non può vedere,

varca i limiti dell'universo.

Cede il passo la mia natura umana,

si allontana da me:

vola in alto, non so più cosa accade,

la mia vista si illumina.

Come si vede, si tratta di una rielaborazione del passo del canto introduttivo alla terza cantica ( $P d$. I 43-93) in cui Dante descrive la sua transumanazione e l'ascesa al Paradiso. Pur non essendoci un preciso corrispettivo letterale, la sequenza della fonte è però, seppure un po' liberamente, ricalcata: il meccanismo di raggi luminosi usato come paragone da Dante ${ }^{44}$ probabilmente incrociato con la prima descrizione del fenomeno fornita da Beatrice, ${ }^{45}$ l'accenno alla possibilità di fissare il sole senza perdere la vista, ralizzabile solo in virtù della peculiare condizione del Paradiso terrestre, ${ }^{46}$ l'allusione al «trasumanar» ${ }^{47}$ $\mathrm{e}$, infine, il movimento inconsapevolmente ascensionale ${ }^{48}$ e la visione totalizzante della luce celeste. ${ }^{49}$ Naturalmente la versione dei Metamorfosi si carica di evidenti per quanto elementari significati simbolici (ad esempio nel contrasto tra "chi non può vedere» e "la mia vista si illumina») del tutto assenti dal testo dantesco: ma, nello stesso tempo, la qualità della rielaborazione, evitando la riattualizzazione perseguita nel primo disco, configura in maniera molto più decisa il testo come una vera e propria riscrittura.

43. Fa eccezione Introduzione, in cui i versi di $P d$. I I-9 sono esattamente trasposti. Di nuovo ci troviamo di fronte ad una scelta speculare rispetto a quella messa in atto in Inferno: là infatti l'Introduzione era un testo sostanzialmente privo di raccordo con quello di Dante e cantato, qui invece si tratta di una citazione letterale eseguita da una voce recitante, a marcare con forza il distacco con i testi che seguiranno.

44. Pd. I 49-5I: «E sì come secondo raggio suole / uscir del primo e risalire in suso, / pur come pelegrin che tornar vuole».

45. Pd. I 9I-93: «Tu non sè in terra, sì come tu credi; / ma folgore, fuggendo il proprio sito, / non corse come tu ch'ad esso riedi».

46. Pd. I 54-57: «e fissi li occhi al sole oltre nostr'uso. / Molto è licito là, che qui non lece / a le nostre virtù, mercé del loco / fatto per proprio de l'umana spece».

47. Pd. I 70-7I: «Trasumanar significar per verba / non si poria [...]».

48. $P d$. I 9I: «Tu non sè in terra, sì come tu credi».

49. Pd. I 58-63: «Io nol soffersi molto, né sì poco, / ch'io nol vedessi sfavillar dintorno, / com' ferro che bogliente esce del foco; / e di sùbito parve giorno a giorno / essere aggiunto, come quei che puote / avesse il ciel d'un altro sole addorno" e 79-8r: "parvemi tanto allor del cielo acceso / de la fiamma del sol, che pioggia o fiume / lago non fece alcun tanto disteso». 
Non molto dissimile, anche se con un allentamento nel rapporto diretto con l'ipotesto, quello che accade nella canzone subito successiva, Cielo della Luna:

L'impotenza dei deboli

si abbandona in voi, lungo strade possibili per raggiungere il cielo.

L’arroganza di chi è cieco e sordo

qui si fermerà, confuso dalle nebbie della luna

la sua forza avrà verso gli inferi

e luce sarà.

Volti diafani e pallidi, specchi della realtà:

l'umiltà di un paradiso spento

si apre intorno a noi.

Lontano dalla luce sulla luna

la felicità senza limiti, mondo intenso di immagini, mondo senza pietà.

Il riferimento agli spiriti beati che non riuscirono a compiere i propri voti a causa della violenza di altri è un dato certo presente ma non rinvia alla dinamica narrativa del passo dantesco di $P d$. III. Persino un elemento figurativo evidentemente derivato dall'originale come quello delle anime percepite da Dante come immagini riflesse in uno specchio o nell'acqua ${ }^{50}$ viene articolato in termini fortemente simbolici, e lo stesso accade anche per le eventuali altre possibili tangenze testuali o di struttura..$^{5 \mathrm{~L}} \mathrm{La}$ chiave della trasposizione lirico-simbolica è dunque quella dominante in Paradiso così come quella dell'invettiva attualizzante lo era in Inferno: di conseguenza si perde quasi completamente il carattere fortemente politico-militante della terza cantica oltre, naturalmente, alla sua portata e complessità teologica. ${ }^{52}$ Per concludere con gli esempi, in Salita a Mercurio il complesso gioco di luci che velano

50. Pd. III IO-2I: "Quali per vetri trasparenti e tersi, / o ver per acque nitide e tranquille, / non sì profonde che i fondi sien persi, / tornan d'i nostri visi le postille / debili sì, che perla in bianca fronte / non vien men forte a le nostre pupille; / tali vid'io più facce a parlar pronte; / per ch'io dentro a l'error contrario corsi / a quel ch'accese amor tra l'omo e 'l fonte. / Sùbito sì com'io di lor m'accorsi, / quelle stimando specchiati sembianti, / per veder di cui fosser, li occhi torsi».

5I. Questo varrà ad esempio nel riferimento all' «umiltà» se è da collegare, come ritengo, alla condizione claustrale di Piccarda e Costanza.

52. Non sarà un caso che il noto tratto dei canti X-XIV, quello dedicato agli spiriti sapienti e alle biografie di san Francesco e san Domenico (oltre che all'ardua spiegazione teologica dell'eccezionale sapienza di Salomone), dove militanza e teologia si intersecano indissolubilmente, verrà reso con un brano strumentale (Il Sole, appunto). 
altre luci evocato da Dante nella descrizione dell'incontro con Giustiniano ${ }^{53}$ si evolve nell'allusione ad una non meglio definita rivelazione ( Verso luce sparsa nel cielo di Mercurio, / la pace cambia volto / la nebbia si dirada quassù»), mentre tutto il lungo discorso dell'imperatore viene condensato nella rielaborazione dell'immagine di $P d$. VI IO6-IO8 ( Stringono gli artigli dell'aquila e strappano / il vello del leone: / l'Impero fa tremare i potenti»). ${ }^{54} \mathrm{Il}$ testo scivola poi verso l'evocazione dell'avvio del canto VII ( $L$ 'armonia tra i beati accenderà / voci bianchi in un coro di luci; / di preziose gemme si fregerà, / come sfere celesti nel cielo»55), richiama la vendetta di Tito sugli Ebrei ("Giusta la vendetta divina del peccato») con rinvio lessicale a ritroso nel canto $\mathrm{VI}^{56}$ ma collocazione congruente rispetto al prosieguo del VII (dove Beatrice appunto risolve il dubbio di Dante circa la conformità di tale punizione a fronte della necessità del sacrificio di Cristo ${ }^{57}$ ), collocazione ribadita dall'allusione al discorso sull'incarnazione del Figlio, sulla crocefissione e sulle modalità con cui si è realizzata la redenzione che ancora Beatrice articola a $P d$. VI 30-I20 ("la croce per salvare, / la duplice natura dell'Uomo; / di misericordia ma anche di giustizia / la nostra redenzione si nutre, / ma non cambia il destino»). Nel finale della canzone questa rete di rinvii si condensa in una considerazione generica sulla concordia che finalmente dovrebbe unire il destino dell'uomo ad un universo fatto di «luce infinita».

Non è il caso di proseguire nell'analisi particolareggiata dei singoli brani: penso si sia infatti capito a sufficienza come Paradiso sia mosso da una strategia di rapporto con l'ipotesto dantesco del tutto diversa da quella di Inferno. Se da un lato, infatti, la rete di allusioni al testo della terza cantica si fa meno scontata, fuggendo dalla citazione memorabile per scegliere la strada della rievocazione puntiforme di specifici passaggi narrativi (talvolta non senza abilità, altre volte con esiti persino un po' criptici), dall'altro si perde quasi completa-

53. Pd. V I33-I37: «Sì come il sol che si cela elli stessi / per troppa luce, come ’l caldo ha róse / le temperanze d'i vapori spessi,/ per più letizia sì mi si nascose / dentro al suo raggio la figura santa». Poco prima Mercurio era stato definito (vv. I28-I29) «[...] la spera / che si vela a' mortai con altrui raggi».

54. In Dante, appunto: «e non l'abbatta esto Carlo novello / coi Guelfi suoi, ma tema de li artigli / ch'a più alto leon trasser lo vello».

55. A essere richiamati sono qui evidentemente il canto e la danza delle anime che come "velocissime faville» (Pd. VII 8) si allontanano da Dante una volta concluso il discorso di Giustiniano, mentre quelle "preziose gemme [...] / come sfere celesti nel cielo» (si sorvoli qui sulla ineleganza del poliptoto, che ci si augura non voglia emulare quelli danteschi così fitti in questo canto) possono essere memoria di $P d$. VI I24-I29 (richiamato anche dalla presenza di «note» e «luce»): «Diverse voci fanno dolci note; / così diversi scanni in nostra vita / rendon dolce armonia tra queste rote. / E dentro a la presente margarita / luce la luce di Romeo, di cui / fu l'ovra grande e bella mal gradita».

56. Pd. VI 92-93: "poscia con Tito a far vendetta corse / de la vendetta del peccato antico».

57. Da $P d$. VII 20-2I ("come giusta vendetta giustamente / punita fosse [...]»), ribadito ai vv. 49-5I ( «Non ti dee oramai parer più forte, / quando si dice che giusta vendetta / poscia vengiata fu da giusta corte») verrà appunto il sintagma "Giusta la vendetta». 
mente l'intento un po' didascalico che caratterizzava il primo disco. Il livello testuale, insomma, dimostra una maggiore raffinatezza e il carattere che ho più volte definito (con un po' di approssimazione, lo ammetto) lirico-simbolico si realizza in conclusione, proprio con il brano finale La Chiesa delle Stelle, in totale libertà rispetto al dettato della Commedia ${ }^{58}$ con il recupero di una dimensione di purificazione e rinascita religiosa che sicuramente rende più generico il nucleo ideologico paradisiaco ma non si può dire che lo tradisca completamente.

La riscrittura dei Metamorfosi si chiude, dopo un altro lasso di tempo non indifferente, con Purgatorio, di cui si danno due versioni molto diverse a seconda che sia stato pubblicato in vinile o in CD (il primo contiene dodici brani mentre il secondo ne ha diciannove, ben sette in più::99 naturalmente terrò conto qui della versione più ampia). Ancora una volta partiamo dall'elenco delle tracce:

Eco Degli Inferi
Catone
Angelo Nocchiero
Negligenti
La Mala Striscia
Porta del Purgatorio
Superbi
Invidiosi
Iracondi
La Chiesa e l'Impero
Accidiosi
La Femmina Balba
Avidi e Prodighi
Golosi
Lussuriosi Purgatorio
Paradiso Terrestre
Beatrice
Il Carro e l'Aquila
E Rinnovato Volo

Rispetto ai due dischi precedenti si registra una drastica diminuzione dei brani strumentali: uno solo, Paradiso Terrestre, presente peraltro solo nella versione in CD. Collegamenti tra brani consecutivi si danno nella sequenza iniziale Catone, Angelo Nocchiero e Negligenti, con evidente funzione di prologo, tra $\mathrm{La}$ Chiesa e l'Impero e Accidiosi e infine tra Paradiso Terrestre, Beatrice e Il Carro e

58. Fatta salva la persino ovvia citazione «Tu sei Dio che muove il cielo e l'altre stelle».

59. I brani in più della versione in CD sono Negligenti, Porta del Purgatorio, La Chiesa e l'Impero, Accidiosi, La Femmina Balba, Paradiso terrestre, Beatrice. Per le due schede tecniche cfr. https://www.discogs.com/it/Metamorfosi-Purgatorio/release/916I746 e https://www. discogs.com/it/Metamorfosi-Purgatorio/release/9161672. 
l'Aquila (a parte Catone, Angelo Nocchiero e Il Carro e l'Aquila sono tutti brani aggiunti nell'edizione ampliata). L'elemento più innovativo, comunque, notevole già sin dal primo scorrere l'elenco dei titoli, è che per la prima volta i Metamorfosi rispettano alla lettera la successione narrativa del poema: non solo ma, come vedremo tra poco, seguono anche con una certa fedeltà la sostanza degli episodi (con un distacco più percepibile in E rinnovato volo, comprensibile però se se ne valuta appunto la collocazione conclusiva) e, spesso, la stessa lettera dei canti. Manca, insomma, ogni tipo di riattualizzazione, com'era in Inferno, o di rilettura lirica, come in Paradiso, e sembra questa volta che sia proprio il racconto di Dante in quanto tale ad aver attratto il gruppo romano.

Entrando più nel dettaglio, andrà intanto segnalato che come già in Paradiso anche in questo terzo disco il brano introduttivo (Eco degli Inferi: il titolo dipende dal fatto che viene ripresa la stessa melodia di Conclusione del primo disco) si caratterizza per l'uso della voce recitante che legge i versi di inizio cantica (qui Pg. I I-I2): la connessione musicale (con Inferno) e strutturale (con Paradiso) è tale quindi da compattare in un insieme l'intera trilogia. A questo prologo segue Catone che si caratterizza rispetto alle strategie testuali utilizzate nei precedenti dischi in maniera molto netta:

"Chi siete voi, anime evase dalla prigione eterna?

Chi vi ha condotto fuori dalla profonda notte inferna?

Ahi, son le leggi dell'abisso o pur del cielo

che le mie porte a voi dannati chiudere non devo?»

«Cerchi de lo dolente regno abbiamo attraversato,

colpa di un solo e triste uomo perso nel peccato.

Spinti da una forza eterna e da una donna pura

nel tuo cospetto siamo giunti privi di paura»

Queste le novità introdotte:

a) il testo è strutturato attraverso un vero e proprio dialogo; ${ }^{60}$

b) non è dato capire chi sta parlando se non si fa riferimento al titolo o, ancora meglio, all'ipotesto, così che una conoscenza di esso appare in qualche modo indispensabile per un pieno apprezzamento dell'operazione condotta, ${ }^{6 \mathrm{I}}$

6o. Qualcosa di simile, dicevo, accadeva anche in Lussuriosi e in Avari di Inferno, ma in quei casi la seconda strofa rappresentava comunque un intervento autoriale, non una vera e propria battuta dialogica come qui. Da notare che in Catone il dialogo è marcato anche a livello musicale col contrasto tra una prima parte fortemente ritmata, basata principalmente su basso, batteria, piano elettrico e hammond e un cantato caratterizzato da una emissione vocale decisamente sostenuta, ed una seconda in cui la base ritmica sparisce completamente e rimangono solo i sintetizzatori, mentre la voce questa volta è tenuta su una timbrica molto più modulata.

6I. Questo non valeva ovviamente per Inferno, dove come ho detto accanto alle innovazioni estranee alla Commedia si registrano esclusivamente riferimenti a canti e versi che possiamo dire appartenere all'enciclopedia diffusa, e neppure in Paradiso, dove il riferimento dantesco non era quasi mai necessario per una comprensione compiuta dei testi. 
c) pur essendo del tutto fedele all'episodio dantesco (mi riferisco ovviamente a Pg. I 40-IO8) e riprendendone talvolta alcuni sintagmi caratteristici, ${ }^{62}$ il testo non è un calco di esso ma ne rappresenta una rielaborazione;

d) manca del tutto ogni possibile riferimento ad una voce autoriale o di commento.

Questi quattro elementi uniti comportano appunto quella forte novità di impostazione che anticipavo poco sopra e che appare più o meno costante in quasi tutti i testi di Purgatorio. In particolare l'assenza di una voce identificabile con quella dell'autore determinerà nel seguito del disco una sovrapposizione tra l'io narrante e Dante che era presente in maniera solo molto episodica nei due dischi precedenti (visto che in Paradiso tale sovrapposizione avveniva a scapito di un'aderenza alla struttura originaria dell'ipotesto). Questo è subito evidente nel brano successivo, Angelo nocchiero:

Misteriose stelle, mare immobile

il cielo immenso su di noi:

fuori dalla nebbia un vascello di anime,

nocchiero è un angelo.

Incredulo io, incredulo,

dopo l'inferno io incredulo.

I due versi iniziali riprendono i primissimi elementi paesaggistici su cui Dante indugiava a $P g$. I 13-27, se le «misteriose stelle» sono, come suppongo, le «quattro stelle / non viste mai fuor ch'a la prima gente» ( $P g$. I 23-24) che il viator si trova a contemplare appena uscito dall'Inferno. Seguono altri due versi che pure se privi di richiami letterali rinviano evidentemente all'episodio dell'arrivo della nave guidata dall'angelo in $P g$. II I3-5I. I due versi finali rappresentano invece un'aggiunta originale ma tutt'altro che incongrua se consideriamo che tutta questa sezione incipitaria (che, come ricordo, è anche musicalmente collegata senza soluzione di continuità) ha la funzione di veicolare il racconto e l'ascoltatore dal clima infernale a quello del secondo regno. Il dato è ancora più evidente con il terzo brano, Negligenti, che in realtà questa volta condensa con estrema libertà un ampio gruppo di canti:

62. Derivano direttamente dal canto l'attacco «Chi siete voi» (Pg. I 40) e il sintagma «prigione eterna» (Pg. I 4I) del primo verso, l'intero secondo verso (che è un collage sintetico di tessere prelevate da $P g$. I 43-45), il sintagma «son le leggi dell'abisso» (Pg. I 46) e la ravvicinata menzione del «cielo» (che sintetizza in una parola Pg. I 47) del terzo verso, mentre il quarto verso è una rielaborazione più libera che però deriva da Dante $(P g$. I 48) almeno l'attacco in "che» e la menzione dei "dannati», nonché «le mie porte» che ormeggia in assonanza «le mie grotte» originario. La seconda strofa si limita invece a rimaneggiare la sostanza della risposta di Virgilio senza riprese puntuali (vv. 52-84), ma con un interessante salto in avanti, visto che l'avvio di essa ricalca senza dubbio Pg. VII 22-23: «Per tutt'i cerchi del dolente regno / [...] son io di qua venuto». Segnalo infine che la presenza di alcuni arcaicismi come «Ahi» e «lo dolente» (peraltro non prelevati direttamente dalla Commedia) connotano evidentemente ancora di più l'operazione in senso, diciamo così, mimeticamente dantesco. 
Prima che sia troppo tardi si risvegliano le anime e volgono lo sguardo all'orizzonte.

Le sette stelle vedono sul ciglio del tramonto;

facili prede fuggono dal tristo indemoniato.

Qui l'elemento più forte, aldilà dell'aderenza generica all'episodio di Pg. II I24-I33 e dell'innovazione paesaggistica delle "sette stelle», ${ }^{63}$ è infatti la trasformazione (diciamo così) di Catone in un "tristo indemoniato", così da sovrapporre (magari un po' incongruamente) l'improvviso dileguarsi delle anime all'uscita di Dante e Virgilio dai gironi infernali.

Il resto del disco alterna la descrizione delle varie cornici purgatoriali ad episodi più specifici ma sempre direttamente evocati dalla cantica. Così il testo di La Mala Striscia prende spunto da Pg. VIII 97-III unendolo con IX I9-33:

La mala striscia infame

rifugge nella selva

e l'aquila mi leva

verso alture incandescenti

e torna luce nei miei occhi spenti

Si copre di silenzio

la valle dei potenti

la testa di un serpente

e poi due angeli dal cielo

impugnano due spade fiammeggianti.

Se infatti «rifugge nella selva» è evidentemente costruito su «fuggì 'l serpente» di VIII I07, la «valle dei potenti» è l'equivalente della "picciola vallea» di VIII 98 e i "due angeli dal cielo" sono i due "astor celesti» che scendono a VIII I04-5 per cacciare il serpente. Proprio l'allusione agli sparvieri avrà poi veicolato ai vv. 3-5 della prima strofa la memoria del sogno dell'aquila in $\mathrm{Pg}$. IX 28-30 che appunto «[...] mi parea che [...] / terribil come folgor discendesse, / e me rapisse suso infino al foco». Insomma, in La Mala Striscia il racconto dei Metamorfosi è davvero e per la prima volta il racconto del poema dantesco, anche se con le ovvie e inevitabili scorciature e adattamenti che il trasferimento ad altra forma comporta. E quasi a siglare l'avvenuto raccordo, la voce di Spitaleri chiude il pezzo recitando le terzine di Pg. VIII 52-57. ${ }^{64}$ Altrettanto

63. Nel Purgatorio infatti, come si ricorderà, c'è una successione tra le quattro stelle che si vedono all'alba e le tre che si vedono al tramonto (cfr. Pg. VIII 88-93). Se si concede un'annotazione a margine, sorprende che i Metamorfosi non abbiano attinto ad un episodio come quello dell'incontro con Casella che si prestava bene a proiezioni, diciamo così, meta-musicali. Ma questo si spiega forse proprio con quella 'oggettivazione' dell'io nel personaggio-Dante di cui dicevo poco prima.

64. Questa riproposta della recitazione diretta dei versi danteschi è un'altra novità di Purgatorio. 
interessante in questo senso è La Femmina Balba, per cui ancora una volta è ben preciso l'aggancio dantesco (qui a Pg. XIX I-33):

E sotto il cielo di Maggior Fortuna, malferma sulle gambe, monca e pallida, parla di sé la strega dei peccati.

Balba è la sua parola e seducente incanta e gli occhi del poeta la dipingono dolce sirena gravida di inganni: di melodie d'amore incanta gli uomini.

E scopre il ventre il mio saggio signore sospinto dalla donna casta e pudica: profonde l'aria un tragico fetore. Custode della cupidigia umana, foriera dei peccati delle genti ignare di seguir percorsi futili, lascia che il sole spenga le tue proposte inutili.

Il dato più interessante qui, andando oltre i soliti precisi echi letterali, ${ }^{65}$ è la ripartizione, diciamo così, tra una prima strofa di tipo diegetico (evidente da quel "gli occhi del poeta» che fa scattare un riferimento metatestuale al Purgatorio stesso) ed una seconda di tipo personale, in cui di nuovo possiamo supporre che l'io si identifichi con Dante. Siamo quindi a un dipresso entro il medesimo meccanismo di Catone, con l'organizzazione strofica che si incarica (insieme ovviamente agli elementi musicali) di indicare le diverse 'voci' chiamate in causa.

Prima di chiudere vorrei aggiungere un'ultima annotazione a rilevare la fedeltà (in questo caso si può spendere la parola) della riscrittura. Se infatti, come dicevo sopra, in Inferno manca sostanzialemente ogni descrizione delle pene a cui sono sottoposti i dannati, in Purgatorio tali descrizioni sono invece sempre presenti e, soprattutto, corrispondono esattamente a quelle dantesche. Dagli invidiosi che portano «Filo di ferro gelido cucito intorno agli occhi, / cilicio, panno ruvido pungente sulla pelle» (Invidiosi) agli iracondi immersi in un «Buio di notte senza stelle, / fumo che penetra negli occhi: / l'ira punita con la cecità» (Iracondi), dagli accidiosi che "Corrono in fretta» (Accidiosi), agli avari, e anzi avari e prodighi, proprio come in Dante ("pregano insieme prodighi ed avari»), che giacciono "Legati mani e piedi, stesi in terra» e così via, tutti questi quadri ripropongono il poema dantesco senza sostanzialmente agire su di esso se non per minimi adattamenti. Il disco realizza dunque e per

65. Si va da «Maggior Fortuna» (in Dante al v. 4), qui risemantizzato, alla descrizione della femmina balba come «malferma sulle gambe, monca e pallida» che riprende i vv. 8-9 danteschi: "ne li occhi guercia, e sovra i piè distorta, / con le man monche, e di colore scialba", a «dolce sirena» (Pg. XIX I9 "Io son", cantava, "io son dolce serena”)) ecc. 
la prima volta una reale trasposizione della parola dantesca nel sistema musicale; e i Metamorfosi, nel loro tragitto decennale dentro la Commedia, partiti dall'idea di una riattualizzazione del poema arrivano così a concretizzare, quasi all'estremo opposto, il progetto di una sua riproposta fedele, riscoprendo così, magari, e sia detto per chiudere con questo paragrafo, in cosa davvero consista l'attualità di Dante.

6. La trilogia dei Metamorfosi non esaurisce però le tangenze dantesche che possiamo rinvenire nel prog italiano. ${ }^{66}$ In linea con quella stagione di recupero del genere che si è sviluppata all'avvio di questo secolo e di cui dicevo all'inizio di queste pagine, proprio negli anni in cui il gruppo romano compiva la propria trilogia uscivano altri tre dischi esplicitamente ispirati a Dante, ovvero Discesa agl'Inferi d'un Giovane Amante de Il Bacio della Medusa (2008), Metafora di un viaggio. Arditi voli di cervelli attenti dei Sezione Frenante (2014) ${ }^{67}$ e Il Pozzo dei Giganti dei Cherry Five (20I5), il primo e l'ultimo per la Black Widow Records, il secondo per la Ma.Ra.Cash. ${ }^{68} \mathrm{Si}$ tratta di tre dischi interessanti per motivi diversi. Il Bacio della Medusa, formatosi nel 2002 da un nucleo di tre giovani musicisti (Simone Cecchini alla voce e chitarra acustica, Federico Caprai al basso e Diego Petrini alla batteria e alle tastiere) a cui si aggregano a partire dall'anno successivo Eva Morelli (flauto e sassofoni), Simone Brozzetti (chitarra elettrica) e Daniele Rinchi (violino e viola), rientra infatti a pieno titolo proprio in quell'ondata neo-prog di cui si parlava nelle

66. Almeno una menzione merita, oltre a quello che si dirà tra poco, il Colossus Project. Ideato dalla rivista finlandese "Colossus» del bassista romano Marco Bernard, il Colossus Project si ripropone di visitare alcuni grandi classici della letteratura affidandone la riscrittura musicale a band prog (nelle sue varie sfumature stilistiche) provenienti da tutto il mondo. Oltre ad avere rielaborato in questo modo il Kalevala, l'Iliade, l'Odissea (e, a partire dal 2oII, anche il Decameron), tra il 2009 e il 2010 sono usciti tre cofanetti, ognuno composto da $4 \mathrm{~cd}$, dedicati nell'ordine a Inferno (2009), Purgatorio (2009) e Paradiso (2010). L'idea era quella di affidare un canto ad ognuno dei gruppi coinvolti e lasciare poi la totale libertà di esecuzione del tema (non mancano quindi brani solo strumentali). Molte delle band presenti in questo progetto sono italiane, naturalmente, ma la natura per così dire 'esterna' di esso (per quanto affascinante), nonché la sua conseguente ed evidente eterogeneità, mi ha consigliato di trascurarne l'analisi in queste pagine. Un profilo piuttosto ampio è comunque in Galvagni, 20I2, p. 65-85.

67. Del disco esiste una seconda versione pubblicata nel 2017 (cfr. https://www.discogs.com/ it/Sezione-Frenante-Metafora-Di-Un-Viaggio-Arditi-Voli-Di-Cervelli-Attenti/release/I0425382), rimixata e che contiene tre bonus track (Fonte, Dieci Giovani, Dieci Giorni, Cento Storie e Carro Di Fuoco) che non hanno rapporto con il progetto originario. Nella versione del 2017 gli elementi più rilevante sono la sostituzione del cantante, con Luciano Degli Alimari (già nella formazione originaria) che subentra a Francesco Nardo, e l'entrata in pianta stabile di Antonio Zullo alle chitarre, che appariva già nella prima versione alla chitarra acustica e che da questo momento sostituisce Doriano Mestriner (che comunque continua ovviamente ad essere accreditato anche in questa versione).

68. Devo la segnalazione del disco de Il Bacio della Medusa a Massimo Gasperini, storico diffusore del prog genovese appunto con la Black Widow Records (la casa discografica e il negozio di dischi), e di quello dei Stazione Frenante a Maurizio Donzella. Ringrazio di cuore entrambi. 
pagine precedenti. ${ }^{69} \mathrm{Al}$ contrario, Cherry Five e Sezione Frenante sono due nomi storici della prima ondata del genere, anche se entrambi con vicende piuttosto burrascose. I primi nascono nel 1973 dall'incontro tra vari musicisti appartenenti alla scena prog romana; ${ }^{70}$ l'anno successivo decidono di cambiare il nome originario, Oliver, in Goblin e registrano un disco che però non viene fatto circolare. A questo punto tre dei membri (Claudio Simonetti, Massimo Morante e Fabio Pignatelli), a cui si unirà il batterista Walter Martino, decidono di proseguire la carriera con la sigla Goblin, componendo nel i975 la fortunatissima colonna sonora di Profondo Rosso di Dario Argento a cui seguirà due anni dopo quella di Suspiria. Il disco del 1974 uscirà invece due anni dopo, omonimo, sotto la sigla Cherry Five (che quindi è un nome in realtà mai realmente utilizzato dalla band) e con i soli Tartarini e Bordini accreditati come membri in copertina. Saranno proprio questi due musicisti che nel 2015 riformeranno la band e registreranno Il Pozzo dei Giganti. ${ }^{71}$ Infine i Sezione Frenante, formatisi a Venezia nel $1974,{ }^{72}$ hanno avuto una fitta attività di concerti come gruppo di supporto dei grandi nomi del prog italiano (dalle Orme al Perigeo e a Biglietto per l'Inferno) tra il 1976 e 1978 quando, come abbiamo visto era successo anche a Il Giro Strano, si sono sciolti senza aver mai inciso un disco. Nel 2006 Mestriner, De Marchi e Casagrande riformano la band insieme al bassista Sandro Bellemo e a Francesco Nardo e qualche anno dopo producono il cd che appunto qui ci interessa e che riprende e riarrangia materiale composto negli anni Settanta..$^{73}$

Anche l'approccio a Dante dei tre gruppi è piuttosto diverso. Se Il Bacio della Medusa prende solo l'episodio di Paolo e Francesca espandendolo e costruendo intorno ad esso un concept ${ }^{74}$ (un po' quindi sulla linea di Caronte dei The Trip), i Cherry Five scelgono tre canti, uno per cantica, creando così una specie di Commedia condensata per esempi e riproponendo dunque in qualche modo e in compendio il modello dei Metamorfosi, ${ }^{75}$ mentre i Sezione

69. Cfr. in proposito il sito del gruppo https://www.ilbaciodellamedusa.net.

70. Il cantante Tony Tartarini aveva già fatto parte (con lo pseudonimo di Toni Gionta) de L'Uovo di Colombo e il batterista Carlo Bordini aveva fatto parte del duo Rustichelli \& Bordini.

7I. Per le varie vicende del gruppo cfr. la scheda su "Italian Prog" http://www.italianprog.com/ it/a cherryfive.htm. Della nuova formazione fanno parte Gianluca De Rossi (tastiere), Ludovico Piccinini (chitarra) e Pino Sallusti (basso).

72. Il nucleo originario includeva, oltre ai già citati Degli Alimari e Mestriner, Mirco De Marchi (tastiere e voce), Moreno Favaretto (basso) e Alessandro Casagrande (batteria).

73. Cfr., oltre al sito del gruppo https://sezionefrenante.eu/, la scheda in "ProgArchives" https:// www.progarchives.com/artist.asp?id=9347 e Italian Prog cit., p. 390.

74. Formula particolarmente cara al gruppo: nel 2012 uscirà infatti un secondo concept, Deus lo vult, dedicato alle Crociate.

75. Interessante anche la decisione di utilizzare strumentazione originale anni Settanta («La musica è il rock progressivo sinfonico degli anni '70, con tanto di strumentazione vintage, soprattutto per quanto riguarda le tastiere (Hammond, Mellotron, Minimoog etc)»; intervista al gruppo pubblicata sul blog "Athos di MAT2020" il 2I giugno 20I5, http://athosenrile. blogspot.com/20I5/o6/cherry-five-il-pozzo-dei-giganti.html), con il risultato di restituire 
Frenante costruiscono pure loro un disco a tema in cui però non c'è focalizzazione su un episodio preciso del poema ma, come indica già il titolo del disco, viene ripreso l'elemento del viaggio (non necessariamente ultraterreno, come vedremo) e lo si utilizza come cornice in cui immettere episodi solo molto sporadicamente implicati con Dante. ${ }^{76}$

Partiamo comunque da Il Bacio della Medusa. Questa la lista delle tracce. ${ }^{77}$

Preludio: il Trapasso

Confessione d'un Amante

La Bestia ed il Delirio

Recitativo: è nel Buio che Risplendono le Stelle

Ricordi del Supplizio

Nostalgia Pentimento e Rabbia

Sudorazione a Freddo sotto il Chiaro di Luna

Melencolia

E Fu allora che dalle Fiamme mi Sorprese una Calda Brezza Celeste

Nosce Te Ipsum: La Bestia Ringhia in Noi

Corale per Messa da Requiem

Epilogo: Conclusione della Discesa agl'Inferi d'un Giovane Amante

Non pochi i brani interamente strumentali, cinque su dodici (La Bestia e il Delirio, Sudorazione e Freddo sotto il Chiaro di Luna, Nosce Te Ipsum: la Bestia Ringhia in Noi e Epilogo: Conclusione della Discesa agl'Inferi d'un Giovane Amante), brani ai quali è peraltro affidata come si può vedere la conclusione del disco. Nell'insieme, comunque, le canzoni nel loro complesso risultano tutte collegate tra loro senza soluzione di continuità, riprendendo dunque dalla tradizione prog anche l'impianto della suite.

Al centro del disco, come dicevo, c'è l'episodio infernale di Francesca da Rimini che ne il gruppo decide di affrontare dalla parte, diciamo così, di Paolo, che diventa quindi l'io narrante. Il racconto prende avvio dalla sua morte (Preludio: il Trapasso), passa poi a raccontare il famigerato episodio del bacio, articolato però come fosse un ricordo ${ }^{78}$ rievocato in un'anticipazione di atmosfera infernale (Confessione d'un Amante). Dopo il primo brano strumentale si apre una sorta di parentesi lirico-meditativa per voce recita-

anche la sonorità della stagione d'esordio del gruppo.

76. Il sito del gruppo parla in effetti di un «lungo viaggio immaginifico che attraversa l'essenza dell'uomo" (https://sezionefrenante.eu/music/; si veda anche l'intervista rilasciata al sito "Yastaradio.com" in occasione della ristampa del 2017 (http://www.yastaradio.com/index. $\mathrm{php} /$ contenuti/recensioni/album-2/yasta-la-vista/I228I-sezione-frenante-metafora-di-unviaggio-revisited).

77. Per la scheda tecnica sul disco cfr. https://www.discogs.com/it/Il-Bacio-Della-Medusa-Discesa-AglInferi-DUn-Giovane-Amante/release/3459329. Dell'album esiste anche un versione in vinile la cui prima facciata si chiude con il pezzo Nostalgia Pentimento e Rabbia

78. Sarà riverbero del famoso esordio di Francesca, in cui si commisera il potere doloroso della memoria (If. V I2I-3: "E quella a me: "Nessun maggior dolore / che ricordarsi del tempo felice / ne la miseria; e ciò sa 'l tuo dottore"»)? 
ta (Recitativo: è nel Buio che Risplendono le Stelle) a cui seguono un secondo momento memoriale in cui si rievoca l'omicidio dei due amanti (Ricordi del Supplizio) ed una specie di confessione postuma che assume anche un po' le vesti dell'epitaffio (Nostalgia Pentimento e Rabbia). A seguito dell'ulteriore brano strumentale viene inserita una parentesi parecchio eccentrica rispetto al racconto tradizionale, in cui l'amore per Francesca viene indicato da Paolo come una specie di momento conclusivo all'insegna della purezza sentimentale di una vita altrimenti trascorsa nella ricerca esclusiva dell'amore sensuale (Melencolia). L'ultimo brano prima del trittico di chiusura di cui dicevo sopra è la sintetica descrizione (due versi) della discesa di Paolo nel girone infernale (E fu allora che dalle Fiamme mi Sorprese una Calda Brezza Celeste).

La rilettura dell'episodio dantesco proposta da Il Bacio della Medusa, spesso attraverso scelte linguistiche volutamente auliche, ${ }^{79}$ è quindi condotta sulla traccia dell'esaltazione dell'irrefrenabile potenza di Amore (bastino in questo senso i versi conclusivi di Nostalgia Pentimento e Rabbia. «Se incontrassi Dio adesso, / gl'invocherei perdono... / Gli chiederei "Conosci la Carne, / hai mai sospirato per Amore?" / "Tu che dettasti le leggi a Mosè, / sei mai stato indotto in tentazione?" / "Tu che hai dimora nell'alto dei Cieli, / sei mai stato arso nel Fuoco Infernale?" ") ma nel contempo, con un movimento contraddittorio evidentemente intenzionale, anche della forza distruttiva che tale potere esercita. Siamo all'interno, insomma, di una lettura che potremmo definire tardoromantica o addirittura, per quanto blandamente, dannunziana (pensando ovviamente alla Francesca da Rimini). Date queste premesse, i calchi propriamente danteschi non possono che essere sporadici: c'è la menzione del libro Galeotto ("Galeotto fu il libro / nell'afa del meriggio», Confessione d'un Giovane Amante ${ }^{80}$ ) e ci sono forse accenni a If. V 107 ( $V$ Viene ogni giorno la nebbia / a mordere le caviglie logore, / stanche d'una sorte dettata / dal folle segno di Caino", Nostalgia Pentimento e Rabbia) e alla "bufera infernal» di If. V 3I («Un Vento caldo intorpidisce le membra... / Nella discesa agl'Inferi d'un giovane amante...»). ${ }^{8 \mathrm{I}} \mathrm{Nel}$ complesso però Dante rimane sostanzialmente solo uno spunto di partenza ampiamente trasfigurato se non addirittura e propriamente tradito.

79. Ne sono sufficiente indizio le frequenti elisioni negli articoli, nelle preposizioni, articolate o meno, nelle forme verbali ecc. (sin dal titolo del disco: agl'Inferi, d'un; e poi sollevar, muovon, gl'inferi, gl'alberi, tormentar, infin, amar e così via), il lessico sempre virato su sinonimi 'alti' e letterari (solo nei primi due brani si registrano giace, arde, volgo, meriggio, ferro - a indicare l'arma dell'omicidio -, spelonche ecc.), il profluvio di maiuscole inserite allo scopo di rendere emblematici alcuni sostantivi (Carne, Bestia, Amore ecc.).

80. Ma è ben dannunziano (seppure non della Francesca), per tornare a quel che dicevo, che l'incontro amoroso avvenga appunto nel «meriggio».

8I. Del resto il disco si chiude proprio con un effetto di sintetizzatore che imita il vento. 
7. È un concept anche Metafora di un viaggio dei Sezione Frenante: e il titolo, come dicevo, chiarisce anche i limiti del rapporto instaurato col poema, rapporto che pure è esplicitato dal gruppo in più di un'occasione. ${ }^{82} \mathrm{La}$ Commedia è infatti assunta come "metafora" di un viaggio che, propriamente e se si permette il gioco di parole, è quello di un'anima e non quello tra le anime. Questo intanto l'elenco delle tracce: ${ }^{83}$

La Quiete In Un Attimo
La Meta Non Trovata
La Meta Non Trovata-Curiosità Di Essere
Attesa
Passaggio
Viscido Ambiente
Pace Immaginata
Quattro Stelle
Note Stonate
Svegliati Luce

Gli unici due brani strumentali sono, in immediata successione, Attesa e Passaggio, peraltro tra i più brevi dell'album (rispettivamente 2' 47" e I' o5") e collegati tra loro senza soluzione di continuità, a marcare un'ideale divisione tra la prima parte del disco, che ha la funzione di prologo, e la seconda che racconta il vero e proprio percorso. Sono collegate anche le subito successive Viscido Ambiente e Pace Immaginata, che come vedremo sono poste in continuità anche dal punto di vista testuale.

Metafora di un viaggio parte dunque con una sequenza di tre brani caratterizzati da liriche molto brevi: ${ }^{84} \mathrm{La}$ Quiete In Un Attimo è il momento in cui il percorso si avvia in termini vagamente onirici («Penso sogno / forse galleggio») che si sostanziano ulteriormente nel «portone alto / bianco immacolato» che ne impedisce il prosieguo in La Meta Non Trovata. Il successivo La Meta Non Trovata-Curiosità di Essere è la breve descrizione dello sconforto di fronte a questa impasse. Questo prologo, che come dicevo è strutturalmente isolato dall'inserimento dei due strumentali subito successivi, non ha evidentemente nulla a che fare con il poema dantesco (se non per quel «portone» metà infernale e metà purgatoriale) e si mantiene linguisticamente su un livello mediano tra ambizioni liricheggianti (ad esempio con il fitto uso di aggettivi

82. Ad esempio nella già citata intervista a Yastaradio.com: «Lentamente prese vita l'idea di completare e mettere per inciso il materiale composto molti anni prima, è nato così "Metafora di un viaggio", concept album ispirato al poetico viaggio di Dante Alighieri». Quanto alla valenza "metaforica", le note interne del cd parlano di «enfatizzazione del viaggio fantasioso che ognuno di noi vorrebbe percorrere» allo scopo di «fare emergere ciò che il pensiero umano è in grado di concepire».

83. Per la schede tecnica cfr. https://www.discogs.com/it/Sezione-Frenante-Metafora-Di-UnViaggio-Arditi-Voli-Di-Cervelli-Attenti/release/5973319.

84. Musiche e testi sono accreditati a Sandro Bellemo. 
e il ricorso a strutture asindetiche) e inattese scivolate verso il colloquiale. ${ }^{85}$ Con Viscido Ambiente e Pace immaginata ci si avvicina un po' di più a Dante, seppure in maniera molto larga. Le due canzoni formano infatti una specie di zona infernale del disco:

\section{Viscido Ambiente}

Un viscido ambiente non vedo la pace

Le gelide ombre non hanno intelletto

Lui c'è non c'è io l'ho dentro

Il nulla ritorna

E così si ripete

L'odio ristagna

e ci si nutre di esso

\section{Pace immaginata}

Un alito di vento interrompe me

Lingue di fuoco spazzano l'oscurità

Cose senza forma

Spariscono nel nulla

Ingoiate da neri flutti

E il silenzio ritorna

Ombre sconosciute

Mi si attaccano come malattie

Che segnano la mia realtà

Che pena

E prego di non stare più qua

Un corridoio

E mi appare qualche cosa che so

Mi spinge

Cammino perché nel fondo del tunnel

Mi riparerò

Se Viscido ambiente riprende, in un contesto complessivamente molto libero, almeno una precisa tessera dantesca là dove si dice che le «ombre non hanno intelletto» ${ }^{86}$ e dunque restituisce senza ambiguità la natura infernale di ciò che viene descritto, Pace immaginata integra questa descrizione con una parte finale in cui il passaggio descritto è evidentemente ricalcato sulla «natural

85. Si pensi a versi come «La vita la morte / lo stress» o a «La materia grigia / non funziona più». In Note Stonate, per fare un altro esempio, si ritrovano, in successione, l'aulicismo "codesto giardino di terra", il sintagma di gusto parlato "sacro poltrire» e il solecismo "Aspira in un futuro migliore»

86. Cfr. appunto If. III i6-I8: «Noi siam venuti al loco ov'i' t'ho detto / che tu vedrai le genti dolorose / c'hanno perduto il ben de l'intelletto». 
burella» che conduce Dante e Virgilio fuori dal primo regno oltremondano alla fine della cantica (If. XXXIV 94-99 e I27-I39). Che l'idea sia proprio quella di ricalcare la successione delle tre cantiche sembrerebbe confermato dalla subito successiva Quattro Stelle, in cui gli intertesti danteschi sono effettivamente ben percepibili:

Quattro stelle salutano chi arriva

Quattro virtù che splendono nel buio

Al primo chiarore la fresca rugiada

Che sparsa sull'erba ci bagna il viso

Il giunco ci cinge i fianchi sudati

Tolte saranno le tracce dell'inferno

Brilla il cielo d'un zaffiro d'oriente

Mentre la luce ci traccia la via

La torrida arsura è caduta da sé

Le membra dolenti si sciolgono ancora

Un timido sole riscalda il mio cuore

Non più prigioniero di tristi presagi

Ricordi lontani di grigi peccati

Fendono l'aria come neri pennacchi

Un ardito volo di anime perse

Costringe lo sguardo ad un cielo diverso

Nuova speranza avvince il mio petto

Impavido nasce un sogno migliore

Chi ha sbagliato si terge la fronte

Risale la china con un sospiro di attesa

Pur con la consueta e prevedibile libertà (ciò di cui si parla è, fuor di metafora, l'uscita dallo stato infernale-depressivo al centro dei due brani precedenti), il testo è intessuto di evidenti echi della Commedia. Nell'avvio sono infatti presenti e percepibili i richiami al primo canto del Purgatorio: oltre alle quattro stelle dei vv. 22-24, già ricordate per i Metamorfosi, troviamo infatti una rielaborazione della parte conclusiva del canto, vv. II 5 -I $33,{ }^{87}$ e il rimaneggiamento del noto v. 13 «Dolce color d'orïental zaffiro». Decisamente slegati dal testo dantesco sono invece i versi successivi, ad eccezione del penultimo ("Chi ha sbagliato si terge la fronte»), in cui credo si possa vedere un richiamo alle sette

87. «L'alba vinceva l'ora mattutina / che fuggia innanzi, sì che di lontano / conobbi il tremolar de la marina. / [...] / Quando noi fummo là 've la rugiada / pugna col sole, per essere in parte / dove, ad orezza, poco si dirada, / ambo le mani in su l'erbetta sparte / soavemente 'l mio maestro pose: / ond'io, che fui accorto di sua arte, / porsi ver' lui le guance lagrimose; / ivi mi fece tutto discoverto / quel color che l'inferno mi nascose. / Venimmo poi in sul lito diserto, / che mai non vide navicar sue acque / omo, che di tornar sia poscia esperto. / Quivi mi cinse sì com'altrui piacque». 
P incise sulla fronte di Dante dall'angelo guardiano della porta purgatoriale a Pg. IX II2-II4 e che spariscono man mano che l'ascesa alle varie cornici procede. Il motivo del viaggio metaforico, comunque, non appare ben innestato sulla memoria dantesca e, alla fine, il testo della canzone si limita a giustapporre i due aspetti senza farli davvero interagire.

Il brano successivo, Note stonate, dovrebbe rappresentare a questo punto la terza ed ultima cantica:

\author{
Nella candida rosa \\ In tanta e perfetta armonia \\ Una nota dà segno di sé \\ Cosa ci sarà laggiù (in basso) \\ Anima mossa da umana virtù \\ Cerca quello che perfetto non è \\ Perché modellarmi nel fango \\ Estrarmi dall'ignoto profondo \\ Pormi in codesto giardino di terra \\ Dopo aver vissuto di $\mathrm{Te}$ \\ Avrei io bisogno di un più alto respiro \\ Non mi basta più un sacro poltrire \\ Tu ci donasti impaziente ragione \\ Che freme davanti all'ignoto sapere \\ Ignoto sapere che sfugge \\ Alla sua (cupida) (avida) mano \\ Dall'alto la sorte la guida \\ Da mille ansie di vita inattesa \\ E con grandi passioni \\ $\mathrm{Si}$ ispira alle muse pagane \\ Sogna grandi avventure \\ Spera in forti emozioni \\ Lui sogna compagne perfette \\ Aspira in un futuro migliore
}

In realtà questa declinazione 'paradisiaca' non è, come si vede, propriamente tale: sarà infatti la canzone successiva, Svegliati Luce, a chiudere il viaggio in una dimensione di ritrovata armonia religiosa («L'umana stirpe / Antiche fazioni / Ormai in pace / Nenie di sogno / Dimore di luce / Che stupisce il Creato») senza peraltro alcun nesso testuale con la Commedia. ${ }^{88}$ Qui semmai si ripropone ancora una volta il tema della ricerca della propria autenticità

88. Se non forse un vago riferimento al passaggio dell'angelo nocchiero che traghetta le anime penitenti dalla foce del Tevere alle spiagge del purgatorio ( $P g$. II 94-IO2) in alcuni versi della seconda strofa («Da mille anni in attesa / Sulle rive del Lete / In attesa del passo / Sul legno divino / Che fila silente /Verso sponde sicure») 
spirituale, più o meno disattesa o ostacolata dalla realtà. E alla fine (o forse proprio come conseguenza di quanto detto) anche i richiami danteschi effettivi sono in sostanza solo due, quello ovviamente ben visibile (ma ribaltato nella sua portata) della "candida rosa» e (ma meno sicuro) la possibilità di leggere dietro il "giardino di terra» un riferimento alla "aiuola che ci fa tanto feroci» di $P d$. XXII I5I. E l'intero disco, nonostante le dichiarazioni, risulta in definitiva fondamentalmente estraneo alla sostanza del poema dantesco (oltre a pagare pegno ad una scrittura testuale non sempre brillantissima).

8. Una maggiore adesione ci si potrebbe invece aspettare da Il Pozzo dei Giganti dei Cherry Five, che già dall'intitolazione delle tracce contiene dei precisi rinvii a tre canti della Commedia: ${ }^{89}$

\section{Il Pozzo dei Giganti (Inferno XXXI) \\ Manfredi (Purgatorio III) \\ a) La forza del guerriero \\ b) Il tempo del destino \\ c) Terra rossa \\ d) Un mondo tra noi due}

Dentro la cerchia antica (Paradiso XVI)

Un elemento di un certo interesse è che in questo disco nessuno dei brani è solo strumentale e, anzi, l'estensione delle liriche e di conseguenza il rilievo del cantato sono decisamente superiori a tutti i casi che abbiamo sin qui incontrato. Certo avrà avuto non poca influenza sulla decisione il fatto che uno dei membri originari alla base della rifondazione della band fosse il cantante, ma comunque si avverte alla lettura dei testi uno sforzo di composizione testuale che è andato di pari passo con quella musicale.

Che l'intento generale sia quello di una riattualizzazione dantesca sul modello di Inferno dei Metamorfosi è dichiarato esplicitamente dal tastierista del gruppo, Gianluca De Rossi:

L'album è chiaramente ispirato ad alcuni episodi della Divina Commedia di Dante, uno per cantica: "Il Pozzo dei Giganti" (che poi ha dato il titolo all'intero lavoro) dal canto XXXI dell'Inferno, dove Dante e Virgilio incontrano i terribili Giganti incastonati dalla cintola in giù in un pozzo posto tra $1^{\prime} 8^{\circ}$ e il $9^{\circ}$ cerchio, in cui i due poeti vengono delicatamente adagiati dal Gigante Anteo, "Manfredi" dal Canto III del Purgatorio, in cui il discendente dell'Imperatore Federico Barbarossa appare come un padre pentito, premuroso e desideroso di riallacciare il legame perduto con la figlia Costanza e prega Dante di fare da tramite, e infine "Dentro la cerchia antica", dal Canto XV del Paradiso, dove la Firenze antica viene vagheggiata come città pudica e onesta in contrapposizione con quella vile e corrotta contemporanea all'autore, molto simile alla società italiana di oggi.

89. Per la scheda tecnica sul disco cfr. https://www.discogs.com/it/Cherry-Five-Il-Pozzo-DeiGiganti/release/7517307. 
Il messaggio quindi è che le tematiche dell'opera dantesca non sono relegate all'epoca in cui venne scritta, ma sono temi universali ancora attuali. $9^{\circ}$

Il valore allegorico è subito ben rilevato nel primo, lungo brano (quasi venticinque minuti) del disco, Il Pozzo dei Giganti, mentre non si può dire lo stesso dell'ipotesto dantesco, che è invece appena alluso in qualche minimo particolare..$^{9 \mathrm{I}}$ I giganti sono incarnazione della superbia («Liberate le mie gambe schiacciate dalla mia superbia») e del potere mondano («troppi giganti fasulli / cresciuti coi soldi degli altri, / troppi giganti vigliacchi / nascondono i piedi di argilla») e rappresentano dunque una facile polarità negativa all'interno del testo. Più interessante semmai è che la canzone preveda un io narrante che si identifica, appunto, con uno dei giganti e che il testo ruoti quasi integralmente intorno al momento della sconfitta e della successiva prigionia agli Inferi. Comunque i due livelli, letterale e allegorico, fanno in realtà un po' fatica a sovrapporsi e alla fin fine il primo risulta decisamente accessorio. Resta infine da segnalare come verso la conclusione de Il Pozzo dei Giganti sia inserito un terzetto di citazioni in latino recitate da Tartarini: la prima è il famoso verso d'avvio di If. XXXIV («Vexilla regis prodeunt Inferni»), la seconda include i vv. I36-I37 del VI libro dell' Eneide («[...] latet arbore opaca / aureus et foliis et lento uimine ramus») e l'ultima i vv. 4I7-4I8 sempre dal VI libro del poema virgiliano («Cerberus haec ingens latratu regna trifauci / personat aduerso recubans immanis in antro»). $\grave{E}$ evidente che questo collage ha lo scopo di fornire una specie di compendio del clima infernale, con una pertinenza variabile rispetto al tema centrale della canzone: ${ }^{92} \mathrm{ma}$ mi sembra soprattutto notevole la proposta di un nesso tra l'aldilà dantesco e quello virgiliano che, in un contesto di cultura pop come quello di cui stiamo discorrendo, possiamo considerare tutt'altro che banale.

Il secondo brano, Manfredi, seppure sempre articolato in una testualità particolarmente ampia, risulta quello più aderente al testo dantesco dell'in-

90. Intervista per "Athos di MAT2020" cit.

9I. L'immagine del "gigante forte e spaventoso / che fa tremare tutto il mondo, / qualunque cosa che si muova e che respiri accanto a me" potrebbe essere debitrice dell'evocazione delle «[...] gran prove / quando i giganti fer paura a' dèi» (vv. 94-95), tanto più che proprio in analogia con Fialte che «[...] tenea soccinto / dinanzi l'altro e dietro il braccio destro / d'una catena che 'l tenea avvinto / dal collo in giù, sì che 'n su lo scoperto / si ravvolgëa infino al giro quinto» (vv. 86-90) si troverà più oltre nel testo «spezzate queste mie catene» (e, ancora più esplicitamente, poco dopo: «Io non combatterò, / non posso usare le mie braccia: / sette giri di catene»). E forse a Nembrot e alle parole che gli rivolge Virgilio («[...] Anima sciocca, / tienti col corno, e con quel ti disfoga / quand'ira o altra passion ti tocca!» vv. 70-72) è possibile rinviare per i versi conclusivi della canzone "posso usare la mia bocca / ma esce solo grande rabbia».

92. Se l'allusione a Lucifero è infatti fortemente avvicinabile, oltre che per l'ovvia compresenza nel poema, per la medesima dinamica visiva per cui una figura gigantesca viene scambiata in un primo tempo per qualcos'altro (mura fortificate nel caso dei Giganti, un mulino nel caso di Lucifero) e Cerbero appare comunque nella Commedia, meno comprensibile è invece il perché dell'evocazione dell'episodio del ramo d'oro. 
tero album. Già la prima sezione, La forza del guerriero, che descrive appunto l'incontro con lo svevo, recupera alcuni spunti abbastanza precisi da $\mathrm{Pg}$. III IO6-I23:

Sì, io lo vedo già,

occhi azzurri e sguardo fiero,

vede intorno a sé

la forza del guerriero,

qualcosa che non va:

l'ambizione che c'è in lui lo perderà.

Grande è la ferita

e grandi i suoi peccati,

non esiste più il fisico perfetto,

ma lo salverà

nel suo cuore gentilezza ed umiltà.

Sì lo salverà

quello che ha nel cuore,

quel senso di umiltà lo salverà.

Ha l'anima ferita

ed è più doloroso

di quello che deturpa la sua faccia.

La rabbia se ne va,

arriva la speranza,

il dolore che c'è in lui non sente più

e quando scorderà

quell'odio che distrugge

case, vite, mogli, figli, affetti e volontà.

Il ritratto recupera come si vede alcuni tratti memorabili del canto: la bellezza di Manfredi, le ferite (anche con la precisa indicazione di quella "che deturpa la sua faccia», con ripresa dunque puntuale del v. Io8 «ma l'un de' cigli un colpo avea diviso»), la menzione dei "grandi... suoi peccati» che è direttamente debitrice del v. I2I («Orribil furon li peccati miei»), il particolare della "gentilezza» e quello ribadito dell' «umiltà» che si ricollegano alla richiesta di perdono pronunciata in punto di morte dal principe svevo. Emerge quindi il ritratto di un principe-guerriero che trova ancor più precisa definizione nella seconda sezione, Il tempo del destino, in cui si rievoca in prima persona (pur senza nominarla esplicitamente) la battaglia di Benevento e la morte in essa di Manfredi (con un accenno ancora una volta al momento del pentimento: "qualcuno forse avrà pietà, / qualcuno poi si pentirà»), e nella terza, Terra rossa, dove si scivola dalla descrizione del campo di battaglia dopo lo scontro all'allusione alla fine della dinastia sveva («Questa terra è persa ormai, / dissolto il sogno di un impero») e ad una sorta di discorso finale che prelude alla quarta sezione, Un mondo tra noi due. In questa ultima parte, infatti, il testo si 
riaggancia sì a Dante ma rielaborando radicalmente la conclusione del canto ${ }^{93}$ grazie all'introduzione (anche qui in prima persona: chi parla, a partire dalla seconda sezione, è sempre Manfredi) della rievocazione del rapporto con la figlia, ovviamente del tutto assente nella Commedia. Proprio questa innovazione ottiene il risultato di far virare la composizione verso una modalità più elegiaca, culminante nella richiesta finale alla figlia di una preghiera,: la stessa, certo, che troviamo appunto nella Commedia, ma che qui diventa momento tutto intimo e perfettamente filiale, appunto («Puoi salvarmi se vuoi, / soltanto tu lo puoi / pregando verso il cielo. / Io ti aspetterò / qui nel mondo tra noi due / e il tempo mio perduto / ritornerà con te»).

Il terzo pezzo, il più breve del disco (ovviamente in senso relativo, visto che sfora ampiamente gli otto minuti), è Dentro la cerchia antica. Interessante qui l'indicazione del XVI del Paradiso come canto di riferimento (anche se il titolo proviene notoriamente da $P d$. XV 97): entro il trittico del cielo di Marte i Cherry Five sembrerebbero dunque tralasciare, infatti, sia le vicende individuali di Cacciaguida e la prima descrizione della città, collocate nel canto XV, sia la tranche autobiografica e profetica, diciamo così, che è centrale nel canto XVII. Detto questo, però, come già per Il Pozzo dei Giganti i rapporti effettivi con Dante sono anche qui particolarmente tenui. L'unico contatto specifico è quello, puramente scenografico e neppure peculiare del canto qui trasposto, della croce luminosa formata dalle anime nel cielo di Marte ("Ognuno è una stella / di luce che brilla / intorno a questa croce di gioia»), mentre per il resto si tratta solo di allusioni generiche ai temi della sete smodata di ricchezza ("Come un cavallo impazzirai, / solo al galoppo ti lancerai, / dietro ad un sogno tu cercherai / lusso e ricchezza intorno a te»), della divisione civile («un mondo corrotto ed ingiusto che / ci porta alla guerra contro tutti») e della doppiezza dei comportamenti umani («Fai attenzione ai maligni che, / gentili e cortesi, sembrano buoni; / restano sempre nell'ambiguità, / ma dentro nascondono un cuore di pietra»). ${ }^{94}$ Il testo nel suo complesso comunque, giusta quando abbiamo letto prima nelle dichiarazioni di De Rossi, opera di fatto una specie di travestimento attualizzante che ricorda molto da vicino quello della trasposizione della prima cantica ad opera dei Metamorfosi.

9. Veniamo ad una possibile sintesi di quello che ho mostrato sin qui. In linea di massima, si sarà notato come l'approccio alla Commedia dantesca sia, nella stragrande maggioranza dei casi qui scorsi, condotto all'insegna di una "attualità" di Dante che è preludio, anche, ad una sostanziale indifferenza per il testo

93. Pg. III I42-I45: «Vedi oggimai se tu mi puoi far lieto, / revelando a la mia buona Costanza / come m'hai visto, e anco esto divieto; / ché qui per quei di là molto s'avanza».

94. Non escluderei per l'inizio della canzone («Niente più pastori qui, / non ci sono greggi») un vago ricordo dell'«ovil di San Giovanni» di $P d$. XVI 25. 
dantesco in quanto tale. Naturalmente questa indifferenza può variare da un minimo a un massimo di lontananza: Inferno e Paradiso dei Metamorfosi riprendono quanto meno la struttura oltremondana del poema, mentre Caronte dei The Trip si limita a recuperare un tassello memorabile della sua iconografia (doppiamente memorabile, perché esplicitamente mediato dalle illustrazioni di Dorè), Metafora di un viaggio dei Sezione Frenante gioca soltanto con l'idea di un percorso vagamente sovrapponibile a quello dantesco e lo stesso Discesa agl'Inferi d'un Giovane Amante de Il Bacio della Medusa si basa sull'inevitabile presenza nella competenza degli ascoltatori di un episodio 'da manuale' come quello dei due amanti riminesi per poi declinarlo in modalità del tutto libere. ${ }^{95}$ Questa "attualità" di Dante, che è poi anche un trasportare Dante in chiave di sensibilità genericamente moderna (il Dante lirico e intimista di Paradiso, quello sensuale e un po' maudit di Discesa agl'Inferi d'un Giovane Amante; o anche il Dante come pretesto per ammonimenti morali contro la superbia e l'avidità di Il Pozzo dei Giganti), prescinde in buona sostanza e di norma da un confronto e di lì da una restituzione del testo dantesco, ma considera la Commedia come un gigantesco segno (in senso proprio semiotico) da giocare nell'ambito di un genere musicale (il prog, appunto) che fa dell'incrocio tra elementi della musica colta ed elementi pop una delle sue marche distintive, al punto che risulta abbastanza arbitrario, nella maggior parte dei casi, parlare di riscritture o di trasposizioni (Dante è il punto di partenza, non molto di più). Colpisce particolarmente, in questo contesto, e concludo, la relativa fedeltà di Purgatorio dei Metamorfosi, unico disco ad affrontare il poema dantesco 'alla lettera' e a proporne una riscrittura in qualche modo alternativa: ma il fatto che si tratti di un tentativo isolato all'interno della stessa trilogia del gruppo romano mi pare sia significativo dell'eccentricità del tentativo. ${ }^{96}$ Il Dante del prog italiano insomma (come forse tutto il Dante veicolato dalla musica pop), e per riprendere una vecchia distinzione di Edoardo Sanguineti che qui può esserci particolarmente utile, rivela alla fine di non essere quasi mai un Dante travestito ma, piuttosto, un Dante in costume.97

95. Lo stesso ragionamento si potrebbe replicare per il brano Paolo e Francesca dei New Trolls.

96. Con una riflessione aggiuntiva da compiere a partire dal fatto che l'unico brano dei Sezione Frenante che dialoga effettivamente col testo dantesco sia proprio la purgatoriale Quattro Stelle e che lo stesso accada con Manfredi dei Cherry Five: non sarà che il secondo regno oltremondano, nella sua problematica collocazione e nella sua assenza di facili suggestioni binarie derivabili invece da Inferno e Paradiso (del tipo buio/luce, male/bene, ma anche corpo/anima, dannazione/beatitudine ecc.), è quello che meno si presta alle semplificazioni attualizzanti e/o simboliche?

97. «Il tutto, finalmente, può anche riassumersi in una formula quasi epigrammatica, per cui un Dante "in travestimento" è il contrario giusto di un Dante "in costume" (Sanguineti,,I989, p. 88). Per una (auto)definizione di «travestimento» in Sanguineti di può vedere la conversazione con Franco Vazzoler pubblicata in appendice a Vazzoler, 2009 (p. I83-2II). 


\section{BiLIOGRAFIA}

Alfano, I. (2004). Fra tradizione colta e popular music: il caso del rock progressivo. Introduzione al genere che sfidò la forma canzone. Roma: Aracne.

Birzer, B. J. (2012, 9 maggio). A Different Kind of Progressive, National Review. (https:// www.nationalreview.com/20I2/os/different-kind-progressive-bradley-j-birzer/).

Ciabattoni, F. (2017). Dante and Italy's Singer-Songwriters, Italian Quarterly, 54, 6I-8O.

Croce, A. (2019). ItalianProg: La guida completa alla musica progressiva italiana degli anni '70. CreateSpace Independent Publishing Platform.

Follero, D. (2009). Concept album. I dischi a tema da Sgt. Pepper's al nuovo millennio. Bologna: Odoya.

Forni, M. (20I0). Lungo le vie del prog: storia del rock progressivo italiano. Personaggi e Opere dal I97I al 20I0. Campobasso: Palladino.

Gaboli, A. e Ottone, G. (2007) Progressive italiano (a cura di Frazz). Firenze: Giunti.

Galvagni, F. (2012). Dante e l'armonia delle sfere (Introduzione di Riccardo Storti, volume corredato da un cd). Milano: Vololibero edizioni.

Marino, A. e Bruno, M. (2015). Terzo grado. Indagine sul pop progressivo italiano. Milano. Tsunami Edizioni.

Mirenzi, F. (1977). Rock progressivo italiano. Dalla PFM agli Area, dal Moog alle Molotov: la colonna sonora degli anni Settanta (2 voll.). Roma: Castelvecchi.

Pardo, P. (20I7, febbraio). Il cantautorato progressive italiano, Blow Up, 225, 40-49, e (marzo 2017) 226, 26-37.

Reynolds, S. (20I0). Post Punk. 1978-I984 (trad. a cura di Michele Piumini, revisione di Anna Mioni, Milano). Isbn Edizioni.

Salari, M. (2018). Rock progressivo italiano: 1980-2013. Roma: Arcana.

Sanguineti, E. (1989). Commedia dell'Inferno. Genova: Costa \& Nola.

Thelen, P. (1995). The World of Magma. Perfect Sound Forever, 8, (http://www.furious. com/perfect/magma.html\#career).

Vazzoler, F. (2009). Il chierico e la scena. Cinque capitoli su Sanguineti e il Teatro. Genova: il melangolo.

Weigel, D. (20I8). Progressive rock: ascesa e caduta di un genere musicale (a cura di Marco Bertoli, con un saggio di Jacopo Tomatis). Torino: EDT. 
